\title{
Error Bound for the Hartree-Fock Energy of Atoms and Molecules
}

\section{Volker Bach}

Institut für Theoretische Physik, ETH Hönggerberg, CH-8093 Zürich, Switzerland

Received October 25, 1991; in revised form January 2, 1992

\begin{abstract}
We estimate the error of the Hartree-Fock energy of atoms and molecules in terms of the one-particle density matrix corresponding to the exact ground state. As an application we show this error to be of order $O\left(Z^{5 / 3-\delta}\right)$ for any $\delta<2 / 21$ as the total nuclear charge $Z$ becomes large.
\end{abstract}

\section{Introduction}

The nonrelativistic quantum mechanical model for an atom $(K=1)$ or molecule is given by the Hamiltonian

$$
H_{N}(\underline{Z}, \underline{R}):=\sum_{i=1}^{N}\left(-\Delta_{i}-\sum_{j=1}^{K} \frac{Z_{j}}{\left|x_{i}-R_{j}\right|}\right)+\sum_{1 \leqq i<j}^{N} \frac{1}{\left|x_{i}-x_{j}\right|},
$$

acting as a self-adjoint operator on a dense domain $D_{N} \subseteq \bigwedge_{i=1}^{N}\left(L^{2}\left(\mathbb{R}^{3}\right) \otimes \mathbb{C}^{q}\right)$. Here we regard the nuclei of charge $Z_{j}$ as pointcharges at fixed positions $R_{j}$, for $1 \leqq j \leqq K$. For the sake of brevity we denote $\underline{Z}:=\left(Z_{1}, \ldots, Z_{K}\right)$ and $\underline{R}$ $:=\left(R_{1}, \ldots, R_{K}\right)$. The nuclei are surrounded by $N$ electrons of $\operatorname{spin} s=\frac{q-1}{2}$, so, in nature $q=2$. We are interested in the ground state energy

$$
E_{Q}(N, \underline{Z}, \underline{R}):=\inf \left\{\left\langle\Psi_{N}\left|H_{N}(\underline{Z}, \underline{R})\right| \Psi_{N}\right\rangle \mid \Psi_{N} \in D_{N},\left\|\Psi_{N}\right\|=1\right\},
$$

which coincides with the bottom of the spectrum of $H_{N}(\underline{Z}, \underline{R})$. (Henceforth $\left\|\Psi_{N}\right\|=1$ is assumed without further notice.) In general, $E_{Q}(N, \underline{Z}, \underline{R})$ is inaccessible to direct computation. Here we are concerned with the asymptotic validity of approximate theories in the limit

$$
Z \rightarrow \infty, \quad N \approx Z, \quad \underline{Z} / Z \text { fixed, } \min _{1 \leqq i<j \leqq K}\left|R_{i}-R_{j}\right| \geqq c Z^{-2 / 3+\varepsilon} .
$$

To leading order $Z^{7 / 3}, E_{Q}$ is given by the Thomas-Fermi energy $E_{\mathrm{TF}}$, as was shown 
by Lieb and Simon [14]. This is followed by the Scott correction (cf. e.g. [10]) of order $Z^{2}$ :

$$
E_{Q}(Z, \underline{Z}, \underline{R})=E_{\mathrm{TF}}(Z, \underline{Z}, \underline{R})+\frac{q}{8} \sum_{j=1}^{K} Z_{j}^{2}+o\left(Z^{2}\right),
$$

a result proved for neutral atoms by Hughes [6], and Siedentop and Weikard $[18,17]$, and for neutral molecules by Ivrii and Sigal [7]. The next correction includes exchange effects and is expected to be of the form $c_{S} Z^{5 / 3}$, where $c_{S}$ is a constant proposed by Dirac [2] and corrected by Schwinger [16]. Fefferman and Seco [4] have announced a proof that

$$
E_{Q}\left(N_{c}, Z\right)=E_{\mathrm{TF}}(1,1) Z^{7 / 3}+\frac{q}{8} Z^{2}+c_{S} Z^{5 / 3}+o\left(Z^{5 / 3}\right)
$$

for atoms binding $N_{c}$ electrons. Here $N_{c}$ denotes the smallest integer such that $E_{Q}\left(N_{c}, Z\right)=\inf _{N \in \mathbb{N}} E_{Q}(N, Z)$. It is known that $Z \leqq N_{c} \leqq Z+o(Z)$ [5]. Also, we indicated the explicit scaling of $E_{\mathrm{TF}}$ in the atomic case.

In this paper we compare $E_{Q}$ with the Hartree-Fock energy

$$
E_{\mathrm{HF}}(N, \underline{Z}, \underline{R}):=\inf \left\{\left\langle\Psi_{N}\left|H_{N}(\underline{Z}, \underline{R})\right| \Psi_{N}\right\rangle \mid \Psi_{N} \in S D_{N} \cap D_{N}\right\},
$$

where $S D_{N}$ are the Slater determinants. We expect, of course, that $E_{\mathrm{HF}}$ already includes the corrections discussed above, i.e. $E_{Q}-E_{\mathrm{HF}}=o\left(Z^{5 / 3}\right)$, where $o\left(Z^{5 / 3}\right)$ might even be $O(1)$. With this goal in mind we first derive an estimate of $E_{Q}-E_{\mathrm{HF}}$ in terms of the one-particle density matrix $\gamma$ (as defined in Sect. 2) corresponding to a ground state $\Psi_{N} \in D_{N}$ of the considered system. Actually, we do not need to use the Schrödinger equation, so $\Psi_{N}$ may just as well be an $\varepsilon$-approximate ground state, i.e.

$$
\left\langle\Psi_{N}\left|H_{N}(\underline{Z}, \underline{R})\right| \Psi_{N}\right\rangle \leqq E_{N}(\underline{Z}, \underline{R})+\varepsilon .
$$

The estimate is as follows

Theorem 1. Let $\gamma$ be the 1-particle density matrix of an $\varepsilon$-approximate ground state $\Psi_{N} \in D_{N}$ of $H_{N}(\underline{Z}, \underline{R})$. Then, for any $0<\delta<1 / 12$,

$$
\left|E_{Q}(N, \underline{Z}, \underline{R})-E_{\mathrm{HF}}(N, \underline{Z}, \underline{R})\right| \leqq d_{\delta} q^{2 / 3} Z N^{2 / 3}\left(\frac{\operatorname{tr}\left\{\gamma-\gamma^{2}\right\}}{N}\right)^{1 / 3-\delta}+\varepsilon,
$$

where $d_{\delta}:=(857.672) \delta^{-1 / 3}$.

This bound is reasonable since, as $\varepsilon \rightarrow 0$, it vanishes if and only if $\gamma$ is a projection or, equivalently, if $E_{Q}(N, \underline{Z}, \underline{R})=\left\langle\Psi_{N}^{S}\left|H_{N}(\underline{Z}, \underline{R})\right| \Psi_{N}^{S}\right\rangle$ for some Slater determinant $\Psi_{N}^{S} \in S D_{N}$. In the next step we estimate $\operatorname{tr}\left\{\gamma-\gamma^{2}\right\}$ in the limit $Z \rightarrow \infty$, using the results of the semiclassical analysis of Ivrii and Sigal [7].

Theorem 2. Consider a molecule of nuclear charges $Z_{j}, 1 \leqq j \leqq K$, with fixed ratios $Z_{j} / Z$, where $Z:=\sum_{j=1}^{K} Z_{j}$ and $K \geqq 1$. Let $\min _{i \neq j}\left\{\left|R_{i}-R_{j}\right|\right\} \geqq c_{1} Z^{-2 / 3+\varepsilon^{\prime}}$ and $Z-c_{2} Z^{1 / 3} \leqq N \leqq Z+c_{3} Z^{5 / 7}$ for some constants $\varepsilon^{\prime}, c_{1}, c_{2}, c_{3}>0$. Let $\gamma$ be the 1-particle density matrix of an $\varepsilon$-approximate ground state $\Psi_{N} \in D_{N}$ of $H_{N}(\underline{Z}, \underline{R})$. Then there exists $c>0$, such that

$$
\operatorname{tr}\left\{\gamma-\gamma^{2}\right\} \leqq c \cdot Z^{5 / 7}
$$

This estimate, inserted in Theorem 1 for arbitrarily small $\varepsilon>0$, proves 
Theorem 3. Consider a molecule of nuclear charges $Z_{j}, 1 \leqq j \leqq K$, with fixed ratios $Z_{j} / Z$, where $Z:=\sum_{j=1}^{K} Z_{j}$ and $K \geqq 1$. Let $\min _{i \neq j}\left\{\left|R_{i}-R_{j}\right|\right\} \geqq c_{1} Z^{-2 / 3+\varepsilon}$ and $Z-c_{2} Z^{1 / 3} \leqq N \leqq Z+c_{3} Z^{5 / 7}$ for some constants $\varepsilon, c_{1}, c_{2}, c_{3}>0$. Then for any $0<\delta<2 / 21$ there exists $c_{\delta}>0$ such that

$$
\left|E_{Q}(N, \underline{Z}, \underline{R})-E_{\mathrm{HF}}(N, \underline{Z}, \underline{R})\right| \leqq c_{\delta} \cdot Z^{5 / 3-\delta} .
$$

We remark that, for $N=N_{c}$, this result is already implicit in [4].

The paper is organized as follows. Section 2 is a collection of definitions and basic properties of fermion density matrices. In Sect. 3 we recall Lieb's extension of the Hartree-Fock variational principle [12], providing a proof which, we think, simplifies the original one. Section 4 contains an estimate of 2-body correlations in terms of $\gamma-\gamma^{2}$. This is the heart of the proof of Theorem 1, given in Sect. 5. The asymptotics of $Z \rightarrow \infty$ is discussed in Sect. 6, leading to the proof of Theorem 2.

\section{Fermion Density Matrices}

We recall definitions and basic properties of fermion density matrices. For

$$
\mathscr{H}:=L^{2}\left(\mathbb{R}^{3}\right) \otimes \mathbb{C}^{q}
$$

let $\mathscr{F}(\mathscr{H})=\bigoplus_{N=0}^{\infty} \mathscr{H}^{(N)}$ be the corresponding fermion Fock space, i.e. $\mathscr{H}^{(0)}=\mathbb{C}$, and $\mathscr{H}^{(N)}=\bigwedge_{i=1}^{N} \mathscr{H}$ (see [20]). The annihilation and creation operators are defined as usual and obey for all $f, g \in \mathscr{H}$ the anticommutation relations

$$
\begin{aligned}
a(f) a^{\dagger}(g)+a^{\dagger}(g) a(f) & =:\left[a(f), a^{\dagger}(g)\right]=\langle f \mid g\rangle, \\
{[a(f), a(g)] } & =\left[a^{\dagger}(f), a^{\dagger}(g)\right]=0 .
\end{aligned}
$$

Given $N$ orthonormal elements $\chi_{1}, \ldots, \chi_{N} \in \mathscr{H}$, we compute

$$
a^{\dagger}\left(\chi_{1}\right) \ldots a^{\dagger}\left(\chi_{N}\right)|0\rangle=(N !)^{-1 / 2} \sum_{\pi}(-1)^{\pi} \chi_{\pi(1)} \otimes \ldots \otimes \chi_{\pi(N)} \in \mathscr{H}^{(N)}
$$

the sum running over all permutations $\pi$ of $(1, \ldots, N)$. These particular wavefunctions are called Slater determinants and we collect them in the set $S D_{N} \subseteq \mathscr{H}^{(N)}$. Given an orthonormal basis $\left\{\varphi_{i}\right\}_{i \in \mathbb{N}}$ of $\mathscr{H}$, we define $a_{i}:=a\left(\varphi_{i}\right)$. Then the hamiltonian (1) is the restriction of

$$
H=\sum_{k, l=1}^{\infty} h_{k ; l} a_{k}^{\dagger} a_{l}+\frac{1}{2} \sum_{k, l, m, n=1}^{\infty} V_{k l ; m n} a_{l}^{\dagger} a_{k}^{\dagger} a_{m} a_{n}
$$

to $\mathscr{H}^{(N)}$. Here we denoted $h_{k ; l}:=\left\langle\varphi_{k}|h| \varphi_{l}\right\rangle$ and $V_{k l ; m n}:=\left\langle\varphi_{k} \otimes \varphi_{l}|V| \varphi_{m} \otimes \varphi_{n}\right\rangle$, with

$$
h:=\left(-\Delta-\sum_{j=1}^{K} \frac{Z_{j}}{\left|x-R_{j}\right|}\right) \otimes 1\left(\sigma_{x}\right), \quad V:=\frac{1}{|x-y|} \otimes 1\left(\sigma_{x}\right) \otimes 1\left(\sigma_{y}\right),
$$

on $\mathscr{H}$ and $\mathscr{H} \otimes \mathscr{H}$, respectively. In particular, for a Slater determinant $\Psi_{N}=a_{1}^{\dagger} \ldots a_{N}^{\dagger}|0\rangle \in S D_{N}$ it is

$$
\left\langle\Psi_{N}|H| \Psi_{N}\right\rangle=\sum_{k=1}^{N} h_{k ; k}+\frac{1}{2} \sum_{k, l=1}^{N}\left(V_{k l ; k l}-V_{k l ; l k}\right) .
$$


More generally, a $p$-body observable $A$ is given in terms of a self-adjoint operator $A^{(p)}$ on $\bigotimes_{i=1}^{p} \mathscr{H}$ by

$$
A:=\frac{1}{p !} \sum_{i_{1}, \ldots, i_{p}} \sum_{j_{1}, \ldots, j_{p}} A_{i_{1} \ldots i_{p} ; j_{1} \ldots j_{p}}^{(p)} a_{i_{p}}^{\dagger} \ldots a_{i_{1}}^{\dagger} a_{j_{1}} \ldots a_{j_{p}} .
$$

Now, let $\rho_{N}$ be an $N$-particle density matrix (N-pdm), i.e. $\rho_{N}=\sum_{i}\left|\Psi_{N, i}\right\rangle \lambda_{i}\left\langle\Psi_{N, i}\right|$ for orthonormal set $\left\{\Psi_{N, i}\right\}_{i \in \mathbb{N}} \subseteq \mathscr{H}^{(N)}$ and nonnegative numbers $0 \leqq \lambda_{i} \leqq 1$, $\sum_{i} \lambda_{i}=1$. The quantum mechanical expectation of the observable $A$ with respect to the state $\rho_{N}$ may then be written as

$$
\begin{aligned}
\langle A\rangle & :=\operatorname{tr}_{N}\left\{A \rho_{N}\right\}=\frac{1}{p !} \operatorname{tr}_{p}\left\{A^{(p)} \rho_{N}^{(p)}\right\} \\
& =\frac{1}{p !} \sum_{i_{1}, \ldots, i_{p}} \sum_{j_{1}, \ldots, j_{p}} A_{i_{1} \ldots i_{p} ; j_{1} \ldots j_{p}}^{(p)}\left\langle a_{i_{p}}^{\dagger} \ldots a_{i_{1}}^{\dagger} a_{j_{1}} \ldots a_{j_{p}}\right\rangle .
\end{aligned}
$$

The reduced density matrices $\rho_{N}^{(p)}, 1 \leqq p \leqq N, \rho_{N}^{(N)} \equiv \rho_{N}$ are determined if (18) holds for all observables $A$ on $\mathscr{H}^{(N)}$ of the form (17):

$$
\rho_{N j_{1} \ldots j_{p} ; i_{1} \ldots i_{p}}^{(p)}=\left\langle a_{i_{p}}^{\dagger} \ldots a_{i_{1}}^{\dagger} a_{j_{1}} \ldots a_{j_{p}}\right\rangle .
$$

Of course, $\rho_{N}^{(p)} \geqq 0$ and $\operatorname{tr}_{p}\left\{\rho_{N}^{(p)}\right\}=N(N-1) \ldots(N-p+1)$. From the fact that $\sum_{k=1}^{\infty} a_{k}^{\dagger} a_{k}$ is the particle number operator, we easily deduce the recursion relation

$$
\rho_{N j_{1} \ldots j_{p} ; i_{1} \ldots i_{p}}^{(p)}=\frac{1}{N-p} \sum_{k=1}^{\infty} \rho_{N j_{1} \ldots j_{p}, k ; i_{1} \ldots i_{p}, k}^{(p+1)} .
$$

We call $\gamma_{\rho}:=\rho_{N}^{(1)}$ the $1-\mathrm{pdm}$ and $\Gamma_{\rho}:=\rho_{N}^{(2)}$ the 2-pdm corresponding to $\rho_{N}$. The expectation value for $H$ can now be written in the general form

$$
\langle H\rangle=\operatorname{tr}_{1}\left\{h \gamma_{\rho}\right\}+\frac{1}{2} \operatorname{tr}_{2}\left\{V \Gamma_{\rho}\right\}
$$

Now, if $\rho_{N}=\left|\Psi_{N}\right\rangle\left\langle\Psi_{N}\right|$ and $\Psi_{N}=a^{\dagger}\left(\chi_{1}\right) \ldots a^{\dagger}\left(\chi_{N}\right)|0\rangle \in S D_{N}$ then one easily checks

$$
\begin{aligned}
\gamma_{\rho} & =\sum_{i=1}^{N}\left|\chi_{i}\right\rangle\left\langle\chi_{i}\right|, \\
\Gamma_{\rho} & =\sum_{i, j=1}^{N}\left|\chi_{i} \wedge \chi_{j}\right\rangle\left\langle\chi_{i} \wedge \chi_{j}\right| \\
& =\left(\gamma_{\rho} \otimes \gamma_{\rho}\right)-\operatorname{Ex}\left(\gamma_{\rho} \otimes \gamma_{\rho}\right),
\end{aligned}
$$

matching (21) with (16). Here,

$$
E x:=\sum_{i, j}\left|\varphi_{i} \otimes \varphi_{j}\right\rangle\left\langle\varphi_{j} \otimes \varphi_{i}\right|
$$

is the exchange operator. Note that $\frac{1}{2}(1-E x)$ is the projection onto $\mathscr{H} \wedge \mathscr{H}$ and it commutes with $\gamma \otimes \gamma$. So, certainly, $(1-E x)(\gamma \otimes \gamma)$ is self-adjoint. Finally, since $0 \leqq \rho_{N k ; k}^{(1)}=\left\langle a_{k}^{\dagger} a_{k}\right\rangle=1-\left\langle a_{k} a_{k}^{\dagger}\right\rangle \leqq 1$ independently of the chosen basis, we have $0 \leqq \gamma_{\rho} \leqq 1$. Note that, apart from the last inequality, we did not use the fermionic 
character of the Fock space $\mathscr{F}(\mathscr{H})$. Also, apart from the separability, we did not use the explicit structure of $\mathscr{H}=L^{2}\left(\mathbb{R}^{3}\right) \otimes \mathbb{C}^{q}$, either. We summarize the properties of $\gamma_{\rho}$ and $\Gamma_{\rho}$ (cf. [1]):

$$
\begin{gathered}
\operatorname{tr}_{1}\left\{\gamma_{\rho}\right\}=N, \quad 0 \leqq \gamma_{\rho} \leqq 1 \\
\left\{\gamma_{\rho}=\gamma_{\rho}^{2},(25)\right\} \Leftrightarrow\left\{\exists \Psi_{N} \in S D_{N}: \rho_{N}=\left|\Psi_{N}\right\rangle\left\langle\Psi_{N}\right|, \gamma_{\rho}=\rho_{N}^{(1)}\right\} \\
\Psi_{N} \in S D_{N}, \rho_{N}=\left|\Psi_{N}\right\rangle\left\langle\Psi_{N}\right| \Rightarrow \Gamma_{\rho}=(1-E x)\left(\gamma_{\rho} \otimes \gamma_{\rho}\right) .
\end{gathered}
$$

\section{Lieb's Variational Principle}

In this section we define the Hartree-Fock variational principle and Lieb's variational principle and show that the latter actually is an extension of the former. Recall the definition (6) of the Hartree-Fock energy $E_{\mathrm{HF}}$ of the considered system:

$$
E_{\mathrm{HF}}(N, \underline{Z}, \underline{R}):=\inf \left\{\left\langle\Psi_{N}\left|H_{N}(\underline{Z}, \underline{R})\right| \Psi_{N}\right\rangle \mid \Psi_{N} \in S D_{N} \cap D_{N}\right\} .
$$

Since the trial functions vary over a smaller set, it clearly holds

$$
E_{Q}(N, \underline{Z}, \underline{R}) \leqq E_{\mathrm{HF}}(N, \underline{Z}, \underline{R}) .
$$

Using the notations and definitions of Sect. 2 and ensuring $\Psi_{N} \in D_{N}$ by $\operatorname{tr}_{1}\{h \gamma\}<\infty$, we may rewrite $E_{\mathrm{HF}}$ as

$$
E_{\mathrm{HF}}(N, \underline{Z}, \underline{R})=\inf \left\{\varepsilon_{\mathrm{HF}}(\gamma) \mid \gamma=\gamma^{\dagger}=\gamma^{2}, \operatorname{tr}_{1}\{\gamma\}=N, \operatorname{tr}_{1}\{h \gamma\}<\infty\right\},
$$

where we defined

$$
\varepsilon_{\mathrm{HF}}(\gamma):=\operatorname{tr}_{1}\{h \gamma\}+\frac{1}{2} \operatorname{tr}_{2}\{V(1-E x)(\gamma \otimes \gamma)\}
$$

The Hartree-Fock variational principle is mathematically inconvenient, because the set $S D_{N}$ has no linear structure. Lieb's crucial observation was, that the condition on $\gamma_{\rho}$ to be induced by some Slater determinant $\Psi_{N} \in S D_{N}$ actually can be dropped [12]. We give an alternative proof of this result which we think is considerably simpler and more constructive than the original one.

Lemma 1. Define $\mathscr{H}, h, V$, and $\varepsilon_{\mathrm{HF}}$ by (11), (15), and (31). Let $0 \leqq \gamma \leqq 1, \operatorname{tr}_{1}\{\gamma\}=N$ be a 1-pdm of finite rank. Then there exists a projection $\hat{\gamma}=\hat{\gamma}^{\dagger}=\hat{\gamma}^{2}, \operatorname{tr}_{1}\{\hat{\gamma}\}=N$, such that

$$
\varepsilon_{\mathrm{HF}}(\gamma) \geqq \varepsilon_{\mathrm{HF}}(\hat{\gamma})
$$

Furthermore, $V>0$ implies the strictness of the inequality (32) unless $\gamma$ is a projection itself.

Proof. We may assume $\varepsilon_{\mathrm{HF}}(\gamma)<\infty$. Working in an eigenvector basis of $\gamma$, we may write

$$
\gamma=\sum_{k=1}^{M}\left|\varphi_{k}\right\rangle \lambda_{k}\left\langle\varphi_{k}\right|, \quad \sum_{k=1}^{M} \lambda_{k}=N, \quad 0<\lambda_{k} \leqq 1, \quad\left\langle\varphi_{k} \mid \varphi_{l}\right\rangle=\delta_{k l}
$$


for some $M<\infty$. Let us abbreviate the diagonal elements $\overline{h_{k}}:=h_{k k}=\left\langle\varphi_{k}|h| \varphi_{k}\right\rangle$ and $\bar{V}_{k l}:=V_{k l ; k l}-V_{k l ; l k}=\left\langle\varphi_{k} \wedge \varphi_{l}|V| \varphi_{k} \wedge \varphi_{l}\right\rangle$. Note that the positivity of $V$ implies $\bar{V}_{k l}>0$ for $k \neq l$, and $\bar{V}_{k k}=0$. One easily checks

$$
\varepsilon_{\mathrm{HF}}(\gamma)=\sum_{k=1}^{M} \lambda_{k} \bar{h}_{k}+\frac{1}{2} \sum_{k, l=1}^{M} \lambda_{k} \lambda_{l} \bar{V}_{k l}
$$

We assume that $M>N$ or, equivalently, $\gamma \neq \gamma^{2}$, for otherwise there is nothing to prove. Then there are at least two eigenvalues $0<\lambda_{p}, \lambda_{q}<1$, say, and we may assume without loss of generality,

$$
\bar{h}_{q}+\sum_{k=1}^{M} \lambda_{k} \bar{V}_{k q} \leqq \bar{h}_{p}+\sum_{k=1}^{M} \lambda_{k} \bar{V}_{k p}
$$

Let $\delta:=\min \left\{\lambda_{p}, 1-\lambda_{q}\right\}>0$ and define

$$
\bar{\gamma}:=\left(\sum_{p, q \neq k=1}^{M}\left|\varphi_{k}\right\rangle \lambda_{k}\left\langle\varphi_{k}\right|\right)+\left|\varphi_{p}\right\rangle\left(\lambda_{p}-\delta\right)\left\langle\varphi_{p}|+| \varphi_{q}\right\rangle\left(\lambda_{q}+\delta\right)\left\langle\varphi_{q}\right| .
$$

By computation one checks that

$$
\varepsilon_{\mathrm{HF}}(\bar{\gamma})-\varepsilon_{\mathrm{HF}}(\gamma)=-\delta\left\{\left(\bar{h}_{p}+\sum_{k=1}^{M} \lambda_{k} \bar{V}_{k p}\right)-\left(\bar{h}_{q}+\sum_{k=1}^{M} \lambda_{k} \bar{V}_{k q}\right)\right\}-\delta^{2} \bar{V}_{p q},
$$

hence, $\varepsilon_{\mathrm{HF}}(\bar{\gamma})-\varepsilon_{\mathrm{HF}}(\gamma)<0$, according to our choice of $\delta$. Furthermore, defining

$$
n(\gamma):=\left|\left\{\lambda_{k} \mid 0<\lambda_{k}<1\right\}\right|,
$$

we observe $n(\bar{\gamma}) \leqq n(\gamma)-1$. After at most $M-N$ iterations of this procedure we obtain a 1-pdm $\hat{\gamma}$ which obeys $\varepsilon_{\mathrm{HF}}(\hat{\gamma})<\varepsilon_{\mathrm{HF}}(\gamma)$ and $n(\hat{\gamma})=0$. But the latter means that $\hat{\gamma}=\hat{\gamma}^{2}$ and, hence, proves the assertion.

Using an approximation argument, it is straightforward to deduce from Lemma 1 the following corollary.

Corollary 1 (Lieb's variational principle).

$$
E_{\mathrm{HF}}(N, \underline{Z}, \underline{R})=\inf \left\{\varepsilon_{\mathrm{HF}}(\gamma) \mid 0 \leqq \gamma \leqq 1, \operatorname{tr}_{1}\{\gamma\}=N, \operatorname{tr}_{1}\{h \gamma\}<\infty\right\} .
$$

Now, we apply Lieb's variational principle to a particular trial 1-pdm. Let $\rho_{N}:=\left|\Psi_{N}\right\rangle\left\langle\Psi_{N}\right|$, where $\Psi_{N}$ is an $\varepsilon$-approximate ground state of the molecule we consider. By Lieb's variational principle it holds

$$
E_{\mathrm{HF}}(N, \underline{Z}, \underline{R}) \leqq \operatorname{tr}_{1}\left\{h \gamma_{\rho}\right\}+\frac{1}{2} \operatorname{tr}_{2}\left\{V(1-E x)\left(\gamma_{\rho} \otimes \gamma_{\rho}\right)\right\} .
$$

Thus we have the following bound:

$$
\begin{aligned}
0 & \geqq E_{Q}(N, \underline{Z}, \underline{R})-E_{\mathrm{HF}}(N, \underline{Z}, \underline{R}) \\
& \geqq \operatorname{tr}_{1}\left\{h \gamma_{\rho}\right\}+\frac{1}{2} \operatorname{tr}_{2}\left\{V \Gamma_{\rho}\right\}-\varepsilon-\operatorname{tr}_{1}\left\{h \gamma_{\rho}\right\}+\frac{1}{2} \operatorname{tr}_{2}\left\{V(1-E x)\left(\gamma_{\rho} \otimes \gamma_{\rho}\right)\right\} \\
& =\frac{1}{2} \operatorname{tr}_{2}\left\{V\left[\Gamma_{\rho}-(1-E x)\left(\gamma_{\rho} \otimes \gamma_{\rho}\right)\right]\right\}-\varepsilon .
\end{aligned}
$$


In fact, the last expression is exactly what we are going to consider in the following section.

\section{Correlation Estimate for Fermions}

In this section we give a lower bound on the truncated 2-pdm of an N-fermion system. The interaction is assumed to factorize into projections in each particle variable.

Assume for the moment that $\rho_{N}^{S}=\left|\Psi_{N}\right\rangle\left\langle\Psi_{N}\right|$ where $\Psi_{N}=a^{\dagger}\left(\chi_{1}\right)$ $\ldots a^{\dagger}\left(\chi_{N}\right)|0\rangle \in S D_{N}$ is a Slater determinant. As mentioned in (27), $\Gamma_{\rho}=\left(\gamma_{\rho} \otimes \gamma_{\rho}\right)-E x\left(\gamma_{\rho} \otimes \gamma_{\rho}\right)$. We expect the N-pdm $\rho_{N}$ we will deal with later on to be very close to such a $\rho_{N}^{S}$, in some appropriate sense. Therefore it is reasonable to call $\Gamma_{\rho}^{T}$, defined by

$$
\Gamma_{\rho}^{T}:=\Gamma_{\rho}-(1-E x)\left(\gamma_{\rho} \otimes \gamma_{\rho}\right)
$$

the truncated 2-pdm. Note that we cannot expect $\Gamma_{\rho}^{T}$ to have a definiteness, for Coleman [1] constructed an N-pdm $\rho_{N}$ with BCS type wavefunctions for which $\Gamma_{\rho}$ and, therefore, also $\Gamma_{\rho}^{T}$ had an eigenvalue of order $N$ and, on the other hand, $\operatorname{tr}_{2}\left\{\Gamma_{\rho}^{T}\right\}=-\operatorname{tr}_{1}\left\{\gamma_{\rho}-\gamma_{\rho}^{2}\right\} \leqq 0$.

In practice, we have nonnegative pair interactions $V \geqq 0$ acting on $\mathscr{H} \otimes \mathscr{H}$ and wish to bound $\operatorname{tr}_{2}\left\{V \Gamma_{\rho}^{T}\right\}$. As shown in Sect. 3 we get upper bounds by means of Lieb's variational principle. Our goal is to obtain lower bounds on $\operatorname{tr}_{2}\left\{V \Gamma_{\rho}^{T}\right\}$, too, at least for suitable $V$. The lower bound we will prove is as follows.

Theorem 4. Let $\mathscr{H}$ be a separable Hilbert space and $\mathscr{F}(\mathscr{H})$ the corresponding fermion Fock space. Let $\rho_{N}$ be an $N$-pdm, and denote the corresponding 1- and 2-pdm by $\gamma$ and $\Gamma$, respectively (see Sect. 2). Let $X=X^{\dagger}=X^{2}$ be an orthogonal projection on $\mathscr{H}$. Then

$$
\operatorname{tr}_{2}\left\{(X \otimes X) \Gamma^{T}\right\} \geqq-\operatorname{tr}_{1}\{X \gamma\} \cdot \min \left\{1,7.554\left[\operatorname{tr}_{1}\left\{X\left(\gamma-\gamma^{2}\right)\right\}\right]^{1 / 2}\right\} .
$$

Proof. For the proof of Theorem 4, an appropriate choice of the orthonormal basis $\left\{\varphi_{i}\right\}_{i \in \mathbb{N}} \subseteq \mathscr{H}$ is crucial. We choose $\left\{\varphi_{i}\right\}_{i \in \mathbb{N}}$ to consist of the eigenfunctions of $\gamma$ : $\gamma \varphi_{i}=\lambda_{i} \varphi_{i}$. For $\lambda_{i}>0, \varphi_{i}$ is called a natural orbital of $\gamma_{\rho}$. Recall that the general property (25) of $\gamma_{\rho}$ implies $\sigma\left(\gamma_{\rho}\right) \backslash\{0\}=\sigma_{\text {disc }}\left(\gamma_{\rho}\right) \backslash\{0\}$ and we obtain $\left\{\varphi_{i}\right\}_{i \in \mathbb{N}}$ by adding some discrete $\mathrm{ON}$-basis of $\operatorname{Ker}\left(\gamma_{\rho}\right)$, whose eigenvalues are set to 0 , to the natural orbitals. Also, we remark that for this particular basis we have

$$
\left\langle a_{k}^{\dagger} a_{m}\right\rangle=\left\langle\varphi_{m}|\gamma| \varphi_{k}\right\rangle=\delta_{m k} \lambda_{k} .
$$

We denote $X_{k m}:=\left\langle\varphi_{k}|X| \varphi_{m}\right\rangle$. We have

$$
\begin{aligned}
\operatorname{tr}_{2} & \left\{(X \otimes X) \Gamma^{T}\right\}=\operatorname{tr}_{2}\{(X \otimes X)[\Gamma-(1-E x)(\gamma \otimes \gamma)]\} \\
& =\sum_{k, l, m, n} X_{k m} X_{l n}\left\{\left\langle a_{l}^{\dagger} a_{k}^{\dagger} a_{m} a_{n}\right\rangle-\left\langle a_{k}^{\dagger} a_{m}\right\rangle\left\langle a_{l}^{\dagger} a_{n}\right\rangle+\left\langle a_{k}^{\dagger} a_{n}\right\rangle\left\langle a_{l}^{\dagger} a_{m}\right\rangle\right\} \\
& =\sum_{k, l, m, n} X_{k m} X_{l n}\left\{\left\langle a_{l}^{\dagger} a_{k}^{\dagger} a_{m} a_{n}\right\rangle\right\}-\sum_{k, l} X_{k k} X_{l l} \lambda_{k} \lambda_{l}+\sum_{k, l}\left|X_{k l}\right|^{2} \lambda_{k} \lambda_{l} .
\end{aligned}
$$


For the sake of comprehensibility we break up the proof into several lemmata. We start with the left part of inequality (43).

\section{Lemma 2.}

$$
\operatorname{tr}_{2}\left\{(X \otimes X) \Gamma^{T}\right\} \geqq-\operatorname{tr}_{1}\{X \gamma\}
$$

Proof of Lemma 2. We follow the usual method of the mean field approximation, known from quantum mechanics. More precisely

$$
\begin{aligned}
\operatorname{tr}_{2}\left\{(X \otimes X) \Gamma^{T}\right\} & \\
= & \sum_{k, l, m, n} X_{k m} X_{l n}\left(\left\langle a_{l}^{\dagger} a_{k}^{\dagger} a_{m} a_{n}\right\rangle-\left\langle a_{k}^{\dagger} a_{m}\right\rangle\left\langle a_{l}^{\dagger} a_{n}\right\rangle\right)+\sum_{k, l}\left|X_{k l}\right|^{2} \lambda_{k} \lambda_{l} \\
= & \left\langle\left[\sum_{k, m} X_{m k}\left(a_{m}^{\dagger} a_{k}-\left\langle a_{m}^{\dagger} a_{k}\right\rangle\right)\right]^{\dagger}\left[\sum_{k, m} X_{m k}\left(a_{m}^{\dagger} a_{k}-\left\langle a_{m}^{\dagger} a_{k}\right\rangle\right)\right]\right\rangle \\
& -\sum_{k, l, m, n} X_{k m} X_{l n}\left[a_{m}, a_{l}^{\dagger}\right]\left\langle a_{k}^{\dagger} a_{n}\right\rangle+\sum_{k, l}\left|X_{k l}\right|^{2} \lambda_{k} \lambda_{l} \\
\geqq & -\sum_{k, l}\left|X_{k l}\right|^{2} \lambda_{k}\left(1-\lambda_{l}\right) \geqq-\sum_{k} X_{k k} \lambda_{k} .
\end{aligned}
$$

Note that in the last line the exchange term $\sum_{k, l}\left|X_{k l}\right|^{2} \lambda_{k} \lambda_{l}$ partially cancels the self energy $\sum_{k} X_{k k} \lambda_{k}$ as long as $\lambda_{l}$ is close enough to 1 . Indeed, this is the main idea for proving Theorem 4 . It suggests to treat large and small $\lambda_{k}$ separately. To this end we fix a number $0<\tau<1$ and introduce the operators

$$
c_{p}:=\sum_{k<\tau} X_{p k} a_{k}, \quad d_{p}:=\sum_{k \geqq \tau} X_{p k} a_{k}
$$

where $k<\tau$ and $k \geqq \tau$ denote $\lambda_{k}<\tau$ and $\lambda_{k} \geqq \tau$, respectively. We compute their anticommutation relations and quadratic expectation values. For all $p, q \in \mathbb{N}$ we have

$$
\begin{aligned}
0 & =\left[c_{p}, c_{q}\right]=\left[c_{p}^{\dagger}, c_{q}^{\dagger}\right]=\left[d_{p}, d_{q}\right]=\left[d_{p}^{\dagger}, d_{q}^{\dagger}\right] \\
& =\left[c_{p}, d_{q}^{\dagger}\right]=\left[c_{p}^{\dagger}, d_{q}\right]=\left[c_{p}, d_{q}\right]=\left[c_{p}^{\dagger}, d_{q}^{\dagger}\right], \\
0 & =\left\langle c_{p} c_{q}\right\rangle=\left\langle c_{p}^{\dagger} c_{q}^{\dagger}\right\rangle=\left\langle d_{p} d_{q}\right\rangle=\left\langle d_{p}^{\dagger} d_{q}^{\dagger}\right\rangle \\
& =\left\langle c_{p}^{\dagger} d_{q}\right\rangle=\left\langle d_{p}^{\dagger} c_{q}\right\rangle=\left\langle c_{p} d_{q}\right\rangle=\left\langle c_{p}^{\dagger} d_{q}^{\dagger}\right\rangle .
\end{aligned}
$$

The nonvanishing contributions are

$$
\begin{aligned}
& {\left[d_{p}^{\dagger}, d_{q}\right]=\sum_{k, l \geqq \tau} X_{k p} X_{q l}\left[a_{k}^{\dagger}, a_{l}\right]=\sum_{k \geqq \tau} X_{k p} X_{q k},} \\
& {\left[c_{p}^{\dagger}, c_{q}\right]=\sum_{k, l<\tau} X_{k p} X_{q l}\left[a_{k}^{\dagger}, a_{l}\right]=\sum_{k<\tau} X_{k p} X_{q k},} \\
& \left\langle d_{p}^{\dagger} d_{q}\right\rangle=\sum_{k, l \geqq \tau} X_{k p} X_{q l}\left\langle a_{k}^{\dagger} a_{l}\right\rangle=\sum_{k \geqq \tau} X_{k p} X_{q k} \lambda_{k}, \\
& \left\langle c_{p}^{\dagger} c_{q}\right\rangle=\sum_{k, l<\tau} X_{k p} X_{q l}\left\langle a_{k}^{\dagger} a_{l}\right\rangle=\sum_{k<\tau} X_{k p} X_{q k} \lambda_{k} .
\end{aligned}
$$


We may rewrite $\operatorname{tr}_{2}\{(X \otimes X) \Gamma\}$ as follows:

$$
\begin{aligned}
\operatorname{tr}_{2} & \{(X \otimes X) \Gamma\} \\
& =\sum_{p, q}\left\langle\left(\sum_{l} X_{p l} a_{l}\right)^{\dagger}\left(\sum_{k} X_{q k} a_{k}\right)^{\dagger}\left(\sum_{m} X_{q m} a_{m}\right)\left(\sum_{n} X_{p n} a_{n}\right)\right\rangle \\
& =\sum_{p, q}\left\langle\left(c_{p}^{\dagger}+d_{p}^{\dagger}\right)\left(c_{q}^{\dagger}+d_{q}^{\dagger}\right)\left(c_{q}+d_{q}\right)\left(c_{p}+d_{p}\right)\right\rangle \\
& =\text { (Main Part) + (Remainder) },
\end{aligned}
$$

where

$$
\mathrm{MP}=\text { Main Part }:=\sum_{p, q}\left\langle d_{p}^{\dagger}\left(c_{q}^{\dagger}+d_{q}^{\dagger}\right) d_{q}\left(c_{p}+d_{p}\right)\right\rangle
$$

and

$$
\begin{aligned}
\mathrm{R}= & \text { Remainder } \\
:= & \sum_{p, q}\left\{\left\langle c_{p}^{\dagger}\left(c_{q}^{\dagger}+d_{q}^{\dagger}\right) d_{q}\left(c_{p}+d_{p}\right)\right\rangle+\left\langle d_{p}^{\dagger}\left(c_{q}^{\dagger}+d_{q}^{\dagger}\right) c_{q}\left(c_{p}+d_{p}\right)\right\rangle\right. \\
& \left.\quad+\left\langle c_{p}^{\dagger}\left(c_{q}^{\dagger}+d_{q}^{\dagger}\right) c_{q}\left(c_{p}+d_{p}\right)\right\rangle\right\} \\
= & \sum_{p, q}\left\{2 \operatorname{Re}\left\langle c_{p}^{\dagger}\left(c_{q}^{\dagger}+d_{q}^{\dagger}\right) d_{q}\left(c_{p}+d_{p}\right)\right\rangle+\left\langle c_{p}^{\dagger}\left(c_{q}^{\dagger}+d_{q}^{\dagger}\right) c_{q}\left(c_{p}+d_{p}\right)\right\rangle\right\} .
\end{aligned}
$$

Now we start proving the right part of (43) in Theorem 4 by estimating the Main Part. In fact, MP is large enough to cancel $\operatorname{tr}_{2}\{(X \otimes X)(1-E x)(\gamma \otimes \gamma)\}$. We claim

\section{Lemma 3.}

$$
\begin{aligned}
M P-\operatorname{tr}_{2} & \{(X \otimes X)(1-E x)(\gamma \otimes \gamma)\} \\
& \geqq-\left(\frac{2}{1-\tau}+\frac{1}{\tau}\right) \cdot \operatorname{tr}_{1}\{X \gamma\} \cdot \operatorname{tr}_{1}\left\{X\left(\gamma-\gamma^{2}\right)\right\} .
\end{aligned}
$$

Proof of Lemma 3. We proceed as in Lemma 2.

$$
\mathrm{MP}=\sum_{p, q}\left\langle\left(c_{q}^{\dagger}+d_{q}^{\dagger}\right) d_{q} d_{p}^{\dagger}\left(c_{p}+d_{p}\right)\right\rangle-\sum_{p, q}\left(\left[d_{p}^{\dagger}, d_{q}\right]\left\langle\left(c_{q}^{\dagger}+d_{q}^{\dagger}\right)\left(c_{p}+d_{p}\right)\right\rangle\right) .
$$

Observe that $\sum_{q}\left\langle d_{q}^{\dagger}\left(c_{q}+d_{q}\right)\right\rangle=\sum_{k \geqq \tau} X_{k k} \lambda_{k}$. So, abbreviating $A:=\sum_{q}\left[d_{q}^{\dagger}\left(c_{q}+d_{q}\right)\right.$ $\left.-\left\langle d_{q}^{\dagger}\left(c_{q}+d_{q}\right)\right\rangle\right]$, we obtain

$$
\begin{aligned}
\mathrm{MP}= & \left\langle A^{\dagger} A\right\rangle+\left(\sum_{k \geqq \tau} X_{k k} \lambda_{k}\right)^{2}-\sum_{k \geqq \tau ; l}\left|X_{k l}\right|^{2} \lambda_{l} \\
\geqq & \left(\sum_{k} X_{k k} \lambda_{k}\right)^{2}-\sum_{k, l}\left|X_{k l}\right|^{2} \lambda_{k} \lambda_{l}-2\left(\sum_{k} X_{k k} \lambda_{k}\right)\left(\sum_{k<\tau} X_{k k} \lambda_{k}\right) \\
& -\sum_{k \geqq \tau ; l}\left|X_{k l}\right|^{2}\left(1-\lambda_{k}\right) \lambda_{l} \\
\geqq & \left(\sum_{k} X_{k k} \lambda_{k}\right)^{2}-\sum_{k, l}\left|X_{k l}\right|^{2} \lambda_{k} \lambda_{l}-\frac{1}{\tau} \sum_{k \geqq \tau ; l}\left|X_{k l}\right|^{2}\left(\lambda_{k}-\lambda_{k}^{2}\right) \lambda_{l} \\
& -\frac{2}{1-\tau}\left(\sum_{k} X_{k k} \lambda_{k}\right)\left(\sum_{k<\tau} X_{k k}\left(\lambda_{k}-\lambda_{k}^{2}\right)\right) .
\end{aligned}
$$


Using $\left|X_{k l}\right|^{2} \leqq X_{k k} X_{l l}$ and letting the sums run over their entire range, we arrive at the assertion.

In the next step we estimate the Remainder. Again we cast this into a lemma. Note that $\left[d_{p}^{\dagger}, d_{p}\right]=\sum_{k \geqq \tau}\left|X_{k p}\right|^{2} \leqq \tau^{-1} \sum_{k \geqq \tau}\left|X_{k p}\right|^{2} \lambda_{k} \leqq \tau^{-1}\left\langle d_{p}^{\dagger} d_{p}\right\rangle$, of which there is no analogue for $\left[c_{p}^{\dagger}, c_{p}\right]$. Thus, one could summarize the strategy of the proof of the following lemma as avoiding anticommutators of type $\left[c_{p}^{\dagger}, c_{p}\right]$ in the estimates.

\section{Lemma 4.}

$$
\begin{aligned}
R \geqq & -\left\{\left(\frac{3}{\tau(1-\tau)}+\frac{3}{\tau^{3 / 2}(1-\tau)^{1 / 2}}\right)\left(\operatorname{tr}_{1}\left\{X\left(\gamma-\gamma^{2}\right)\right\}\right)^{1 / 2}\right. \\
& \left.+\frac{2}{\tau^{1 / 2}(1-\tau)^{1 / 2}}\left(1+\frac{1}{\tau(1-\tau)} \operatorname{tr}_{1}\left\{X\left(\gamma-\gamma^{2}\right)\right\}\right)^{1 / 2}\right\} \\
& \cdot \operatorname{tr}_{1}\{X \gamma\} \cdot\left(\operatorname{tr}_{1}\left\{X\left(\gamma-\gamma^{2}\right)\right\}\right)^{1 / 2}
\end{aligned}
$$

Proof of Lemma 4. First observe that by expanding and relabelling

$$
\begin{aligned}
\sum_{p, q}\left\langle c_{p}^{\dagger}\left(c_{q}^{\dagger}+d_{q}^{\dagger}\right) c_{q}\left(c_{p}+d_{p}\right)\right\rangle= & \sum_{p, q}\left(\left\langle c_{p}^{\dagger} c_{q}^{\dagger} c_{q} c_{p}\right\rangle+\left\langle c_{p}^{\dagger} d_{q}^{\dagger} c_{q} d_{p}\right\rangle\right. \\
& \left.+2 \operatorname{Re}\left\langle c_{p}^{\dagger} d_{q}^{\dagger} c_{q} c_{p}\right\rangle\right) .
\end{aligned}
$$

Hence, after some algebra, anticommutations, and dropping of vanishing expectation values,

$$
\begin{aligned}
R= & \sum_{p, q}\left(\left\langle c_{p}^{\dagger} c_{q}^{\dagger} c_{q} c_{p}\right\rangle+4 \operatorname{Re}\left\langle c_{p}^{\dagger} d_{q}^{\dagger} c_{q} c_{p}\right\rangle+2\left\langle c_{p}^{\dagger} d_{q}^{\dagger} d_{q} c_{p}\right\rangle\right) \\
& +\sum_{p, q}\left\langle c_{p}^{\dagger} d_{p} d_{q}^{\dagger} c_{q}\right\rangle-\sum_{p, q}\left[d_{q}^{\dagger}, d_{p}\right]\left\langle c_{p}^{\dagger} c_{q}\right\rangle \\
& +\sum_{p, q} 2 \operatorname{Re}\left\langle c_{p}^{\dagger} d_{q} d_{p} d_{q}^{\dagger}\right\rangle+2 \operatorname{Re}\left\langle\left(\sum_{p} c_{p}^{\dagger} d_{p}\right)\left(\sum_{p} c_{p}^{\dagger} d_{p}\right)\right\rangle .
\end{aligned}
$$

We estimate the above sum term by term, merely using the Cauchy-Schwarz inequality $|\langle A B\rangle|^{2} \leqq\left\langle A A^{\dagger}\right\rangle\left\langle B^{\dagger} B\right\rangle$. But before doing so, let us single out an estimate which will be used over and over again. Written in a somewhat redundant way, it holds

$$
\left|\sum_{p, q}\left\langle c_{p}^{\dagger} d_{q}^{\dagger} d_{q} c_{p}\right\rangle\right|+\left|\sum_{p, q}\left\langle c_{p}^{\dagger} d_{q} d_{q}^{\dagger} c_{p}\right\rangle\right| \leqq \frac{1}{\tau(1-\tau)}\left(\sum_{k} X_{k k} \lambda_{k}\right)\left(\sum_{k} X_{k k}\left(\lambda_{k}-\lambda_{k}^{2}\right)\right),
$$

because the left-hand side of (63) equals

$$
\begin{aligned}
\sum_{p, q}\left\langle c_{p}^{\dagger} c_{p}\right\rangle\left[d_{q}^{\dagger}, d_{q}\right] & =\left(\sum_{k<\tau} X_{k k} \lambda_{k}\right)\left(\sum_{k \geqq \tau} X_{k k}\right) \\
& \leqq \frac{1}{\tau(1-\tau)}\left(\sum_{k<\tau} X_{k k}\left(\lambda_{k}-\lambda_{k}^{2}\right)\right)\left(\sum_{k \geqq \tau} X_{k k} \lambda_{k}\right)
\end{aligned}
$$


Hence, completing the square and using (63), the sum in the right-hand side of (60) is bounded from below by

$$
\begin{aligned}
& \sum_{p, q}\left(\left\langle c_{p}^{\dagger} c_{q}^{\dagger} c_{q} c_{p}\right\rangle-4\left\langle c_{p}^{\dagger} c_{q}^{\dagger} c_{q} c_{p}\right\rangle^{1 / 2}\left\langle c_{p}^{\dagger} d_{q}^{\dagger} d_{q} c_{p}\right\rangle^{1 / 2}+2\left\langle c_{p}^{\dagger} d_{q}^{\dagger} d_{q} c_{p}\right\rangle\right) \\
& \quad \geqq-2 \sum_{p, q}\left\langle c_{p}^{\dagger} d_{q}^{\dagger} d_{q} c_{p}\right\rangle \\
& \quad \geqq-\frac{2}{\tau(1-\tau)}\left(\sum_{k} X_{k k}\left(\lambda_{k}-\lambda_{k}^{2}\right)\right)\left(\sum_{k} X_{k k} \lambda_{k}\right) .
\end{aligned}
$$

The first sum in (61) is obviously nonnegative and the second sum is explicitly computable. After doing the $p, q$ summation the second sum in (61) yields

$$
-\sum_{k \geqq \tau} \sum_{l<\tau}\left|X_{k l}\right|^{2} \lambda_{l} \geqq-\frac{1}{\tau(1-\tau)}\left(\sum_{k} X_{k k}\left(\lambda_{k}-\lambda_{k}^{2}\right)\right)\left(\sum_{k} X_{k k} \lambda_{k}\right) .
$$

The estimate on the first sum in (62) goes as follows:

$$
\begin{aligned}
\sum_{p, q} 2 \operatorname{Re}\left\langle c_{p}^{\dagger} d_{q} d_{p} d_{q}^{\dagger}\right\rangle & \geqq-2 \sum_{p, q}\left(\left\langle c_{p}^{\dagger} d_{q} d_{q}^{\dagger} c_{p}\right\rangle^{1 / 2}\left\langle d_{q} d_{p}^{\dagger} d_{p} d_{q}^{\dagger}\right\rangle^{1 / 2}\right) \\
& \geqq-2\left(\sum_{p, q}\left\langle c_{p}^{\dagger} d_{q} d_{q}^{\dagger} c_{p}\right\rangle\right)^{1 / 2}\left(\sum_{p, q}\left\langle d_{q} d_{p}^{\dagger} d_{p} d_{q}^{\dagger}\right\rangle\right)^{1 / 2}
\end{aligned}
$$

Now, the first factor is taken care of by (63) and in the second factor we again anticommute $d_{p}^{\dagger}$ and $d_{p}$ and use $\left\langle d_{q} d_{q}^{\dagger}\right\rangle=\left[d_{q}^{\dagger}, d_{q}\right]-\left\langle d_{q}^{\dagger} d_{q}\right\rangle$. Hence we obtain

$$
\begin{aligned}
\sum_{p, q}\left\langle d_{q} d_{p}^{\dagger} d_{p} d_{q}^{\dagger}\right) & \leqq\left(\sum_{p}\left[d_{p}^{\dagger}, d_{p}\right]\right)\left(\sum_{q}\left(\left[d_{q}^{\dagger}, d_{q}\right]-\left\langle d_{q}^{\dagger} d_{q}\right\rangle\right)\right) \\
& =\left(\sum_{k \geqq \tau} X_{k k}\right)\left(\sum_{k \geqq \tau} X_{k k}\left(1-\lambda_{k}\right)\right) \\
& \leqq \frac{1}{\tau^{2}}\left(\sum_{k} X_{k k} \lambda_{k}\right)\left(\sum_{k} X_{k k}\left(\lambda_{k}-\lambda_{k}^{2}\right)\right) .
\end{aligned}
$$

The above product therefore leads to the inequality

$$
\sum_{p, q} 2 \operatorname{Re}\left\langle c_{p}^{\dagger} d_{q} d_{p} d_{q}^{\dagger}\right) \geqq-\frac{2}{\tau^{3 / 2}(1-\tau)^{1 / 2}}\left(\sum_{k} X_{k k} \lambda_{k}\right)\left(\sum_{k} X_{k k}\left(\lambda_{k}-\lambda_{k}^{2}\right)\right) .
$$

It remains to estimate $2 \operatorname{Re}\left\langle\left(\sum_{p} c_{p}^{\dagger} d_{p}\right)^{2}\right\rangle$ which will actually give the leading error term. As the way we write this term suggests, we use the Cauchy-Schwarz inequality on the embraced operator, yielding the lower bound

$$
-2\left(\sum_{p, q}\left\langle c_{p}^{\dagger} d_{p} d_{q}^{\dagger} c_{q}\right\rangle\right)^{1 / 2}\left(\sum_{p, q}\left\langle d_{q}^{\dagger} c_{q} c_{p}^{\dagger} d_{p}\right\rangle\right)^{1 / 2}
$$

Now, observe that

$$
\begin{aligned}
\sum_{p, q}\left\langle c_{p}^{\dagger} d_{p} d_{q}^{\dagger} c_{q}\right\rangle & \leqq\left(\sum_{p}\left\langle c_{p}^{\dagger} d_{p} d_{p}^{\dagger} c_{p}\right\rangle^{1 / 2}\right)^{2} \\
& \leqq\left(\sum_{p}\left\langle c_{p}^{\dagger} c_{p}\right\rangle^{1 / 2}\left[d_{p}^{\dagger}, d_{p}\right]^{1 / 2}\right)^{2} \\
& \leqq \sum_{p, q}\left\langle c_{p}^{\dagger} c_{p}\right\rangle\left[d_{q}^{\dagger}, d_{q}\right]
\end{aligned}
$$


so, again, (63) applies. After an anticommutation, a similar argument is used to estimate the other factor

$$
\begin{aligned}
\sum_{p, q}\left\langle d_{q}^{\dagger} c_{q} c_{p}^{\dagger} d_{p}\right\rangle & =\sum_{p, q}\left(\left\langle d_{q}^{\dagger} d_{p}\right\rangle\left[c_{p}^{\dagger}, c_{q}\right]\right)-\sum_{p, q}\left\langle c_{p}^{\dagger} d_{q}^{\dagger} d_{p} c_{q}\right\rangle \\
& \leqq \sum_{k \geqq \tau} \sum_{l<\tau}\left|X_{k l}\right|^{2} \lambda_{k}+\sum_{p, q}\left\langle c_{p}^{\dagger} d_{q}^{\dagger} d_{q} c_{p}\right\rangle \\
& \leqq\left(\sum_{k} X_{k k} \lambda_{k}\right)\left[1+\frac{1}{\tau(1-\tau)}\left(\sum_{k} X_{k k}\left(\lambda_{k}-\lambda_{k}^{2}\right)\right)\right] .
\end{aligned}
$$

Lemma 4 is proved by collecting all these estimates.

Taking into account all the estimates from Lemma 2, 3 and 4 and denoting $\operatorname{tr}_{1}\left\{X\left(\gamma-\gamma^{2}\right)\right\}=: a^{2}, \operatorname{tr}_{1}\{X \gamma\}=: b$ and $g^{-1}:=\tau(1-\tau)$, we arrive at

$$
\begin{aligned}
& \operatorname{tr}_{2}\left\{(X \otimes X) \Gamma^{T}\right\} \\
& \quad \geqq-b \cdot \min \left\{1, a\left[\left(2 \tau g+\frac{1}{\tau}+3 g+\frac{2}{\tau} g^{1 / 2}\right) a+2 g^{1 / 2}\left(1+g a^{2}\right)^{1 / 2}\right]\right\} .
\end{aligned}
$$

We just describe in words how to get from here to the final inequality. Note that the term in brackets, let us call it $F(a, \tau)$, is monotonically increasing in $a$. Also we only consider the range of $a$, where $a F(a, \tau) \leqq 1$. This leads to the range $0 \leqq a \leqq a_{0}(\tau)$ for an explicitly given function $a_{0}(\tau)$. But then $a F(a, \tau) \leqq a F\left(a_{0}(\tau), \tau\right)=a a_{0}^{-1}(\tau)$ and it remains to optimize with respect to $\tau$, which gives $\tau_{\text {opt }}=0.533$ and $a_{0}^{-1}\left(\tau_{\text {opt }}\right)=7.554$, and the above inequality reduces to the desired result.

\section{Error Bound for the Hartree-Fock Energy}

The next task we undertake is the application of the fairly abstract result of Sect. 4 to the Coulomb interaction in atoms and molecules.

5.1. Application of the Correlation Estimate. As explained in Sect. 3 we have

$$
0 \geqq E_{Q}(N, \underline{Z}, \underline{R})-E_{\mathrm{HF}}(N, \underline{Z}, \underline{R}) \geqq \frac{1}{2} \operatorname{tr}_{2}\left\{V \Gamma_{\rho}^{T}\right\}-\varepsilon,
$$

where $\Gamma_{\rho}^{T}$ is the truncated 2-pdm of an N-pdm $\rho_{N}=\left|\Psi_{N}\right\rangle\left\langle\Psi_{N}\right|, \Psi_{N} \in D_{N}$ being an $\varepsilon$-approximate ground state of the molecule in question. $V:=$ $\frac{1}{|x-y|} \otimes 1\left(\sigma_{x}\right) \otimes 1\left(\sigma_{y}\right)$ is the Coulomb interaction on $\mathscr{H} \otimes \mathscr{H}$. We use the decomposition

$$
\frac{1}{|x-y|}=\frac{1}{\pi} \int d^{3} z \int_{0}^{\infty} \frac{d r}{r^{5}} \chi_{B(r, z)}(x) \chi_{B(r, z)}(y)
$$

which was introduced by Fefferman and de la Llave [3]. Here, $\chi_{B(r, z)}$ is the characteristic function of $\left\{x \in \mathbb{R}^{3}|| x-z \mid \leqq r\right\}$. We denote the multiplication 
operator on $\mathscr{H}$ corresponding to $\chi_{B(r, z)} \otimes 1(\sigma)$ by $X_{(r, z)}$. Note that clearly $X_{(r, z)}=X_{(r, z)}^{+}=X_{(r, z)}^{2}$.

Furthermore we define

$$
\begin{aligned}
\rho(x) & :=\sum_{\sigma=1}^{q} \gamma_{\rho}(x, \sigma \mid x, \sigma), \\
\rho_{T}(x) & :=\sum_{\sigma=1}^{q}\left\{\gamma_{\rho}(x, \sigma \mid x, \sigma)-\gamma_{\rho}^{2}(x, \sigma \mid x, \sigma)\right\},
\end{aligned}
$$

so $\rho$ is the one-particle density of the $\varepsilon$-approximate ground state $\Psi_{N}$ and we call $\rho_{T}$ the truncated one-particle density. With the above notations we can prove the following estimate.

Lemma 5. Assume (15), (75) and (76). For any $0<\delta \leqq 5 / 24$ it holds

$$
\frac{1}{2} \operatorname{tr}_{2}\left\{V \Gamma_{\rho}^{T}\right\} \geqq-c_{\delta}\left(\int \rho^{5 / 3}(x) d^{3} x\right)^{1 / 2}\left(\int \rho(x) d^{3} x\right)^{1 / 2}\left(\frac{\int \rho_{T}(x) d^{3} x}{\int \rho(x) d^{3} x}\right)^{1 / 3-\delta}
$$

where $c_{\delta}:=(185.417)(7.554)^{2 / 3} \delta^{-1 / 3}$.

Proof. The first step consists in applying Theorem 4 to $X:=X_{(r, z)}$ and superimposing all the obtained estimates.

$$
\begin{aligned}
\frac{1}{2} \operatorname{tr}_{2}\left\{V \Gamma_{\rho}^{T}\right\}= & \frac{1}{2 \pi} \int d^{3} z \int_{0}^{\infty} \frac{d r}{r^{5}} \operatorname{tr}_{2}\left\{\left(X_{(r, z)} \otimes X_{(r, z)}\right) \Gamma_{\rho}^{T}\right\} \\
\geqq & -\frac{1}{2 \pi} \int d^{3} z \int_{0}^{\infty} \frac{d r}{r^{5}} \operatorname{tr}_{1}\left\{X_{(r, z)} \gamma_{\rho}\right\} \\
& \cdot \min \left\{1, \alpha\left(\operatorname{tr}_{1}\left\{X_{(r, z)}\left(\gamma_{\rho}-\gamma_{\rho}^{2}\right)\right\}\right)^{1 / 2}\right\} \\
= & -\frac{1}{2 \pi} \int d^{3} z \int_{0}^{\infty} \frac{d r}{r^{5}}\left(\int_{|x-z| \leqq r} \rho(x) d^{3} x\right) \\
& \cdot \min \left\{1, \alpha\left(\int_{|x-z| \leqq r} \rho_{T}(x) d^{3} x\right)^{1 / 2}\right\} \\
\geqq & -\frac{1}{2 \pi} \int d^{3} z\left\{\int_{0}^{R(z)} \frac{d r}{r^{5}} \alpha\left(\int_{|x-z| \leqq r} \rho(x) d^{3} x\right)\left(\int_{|x-z| \leqq r} \rho_{T}(x) d^{3} x\right)^{1 / 2}\right. \\
& \left.+\int_{R(z)}^{\infty} \frac{d r}{r^{5}}\left(\int_{|x-z| \leqq r} \rho(x) d^{3} x\right)\right\},
\end{aligned}
$$

for any measurable choice of $R(z)$, with $\alpha:=7.554$. We proceed analogously to Lieb [8] introducing the Hardy-Littlewood maximal functions of $\rho$ and $\rho_{T}$.

$$
\begin{aligned}
M(z) & :=\sup _{r>0}\left\{\frac{3}{4 \pi r^{3}}\left(\int_{|x-z| \leqq r} \rho(x) d^{3} x\right)\right\}, \\
M_{T}(z) & :=\sup _{r>0}\left\{\frac{3}{4 \pi r^{3}}\left(\int_{|x-z| \leqq r} \rho_{T}(x) d^{3} x\right)\right\} .
\end{aligned}
$$


Recall that in general, if $f \in L^{1}\left(\mathbb{R}^{3}\right)$, then its maximal function $M_{f}(z):=$ $\sup _{r>0}\left\{3\left(4 \pi r^{3}\right)^{-1}\left(\int_{|x-z| \leqq r}|f(x)| d^{3} x\right)\right\}<\infty$ on $\mathbb{R}^{3}$ a.e. and fulfills the maximal inequality

$$
\int M_{f}^{p}(z) d^{3} z \leqq \frac{96}{\pi} \frac{p}{p-1} \int|f(z)|^{p} d^{3} z
$$

for all $p>1$ (cf. [19], pp. 58). By means of $M$ and $M_{T}$ we find for any $q>1 / 3$

$$
\begin{aligned}
& \int_{0}^{R(z)} \frac{d r}{r^{5}}\left(\int_{|x-z| \leqq r} \rho(x) d^{3} x\right)\left(\int_{|x-z| \leqq r} \rho_{T}(x) d^{3} x\right)^{q} \\
& \quad \leqq\left(\frac{4 \pi}{3}\right)^{1+q} M(z) M_{T}^{q}(z) \int_{0}^{R(z)} r^{3 q-2} d r \\
& =\left(\frac{4 \pi}{3}\right)^{1+q} \frac{R^{3 q-1}(z)}{3 q-1} M(z) M_{T}^{q}(z)
\end{aligned}
$$

and similarly

$$
\int_{R(z)}^{\infty} \frac{d r}{r^{5}}\left(\int_{|x-z| \leqq r} \rho(x) d^{3} x\right) \leqq \frac{4 \pi}{3} \frac{M(z)}{R(z)},
$$

on $\mathbb{R}^{3}$ a.e. Thus we obtain

$$
\begin{aligned}
& \int_{0}^{\infty} \frac{d r}{r^{5}}\left(\int_{|x-z| \leqq r} \rho(x) d^{3} x\right) \min \left\{1, \alpha\left(\int_{|x-z| \leqq r} \rho_{T}(x) d^{3} x\right)^{1 / 2}\right\} \\
& \quad \leqq \frac{4 \pi}{3} M(z)\left\{\frac{c_{q}}{3 q-1} M_{T}^{q}(z) R^{3 q-1}(z)+R^{-1}(z)\right\},
\end{aligned}
$$

where $c_{q}=(4 \pi / 3)^{q} \alpha$. An optimization yields $R(z)=c_{q}^{-1 / 3 q} M_{T}^{-1 / 3}(z)$ and bounds the right-hand side of the inequality above by

$$
\frac{12 \pi q}{3(3 q-1)} c_{q}^{1 / 3 q} M(z) M_{T}^{1 / 3}(z)
$$

Observe that there is no dependence of the estimate (86) on $q$ apart from an overall constant. In this respect, a substitution of $\left.\operatorname{tr}_{1}\left\{X\left(\gamma-\gamma^{2}\right)\right\}\right)^{1 / 2}$ by $\operatorname{tr}_{1}\left\{X\left(\gamma-\gamma^{2}\right)\right\}$ in Theorem 4 would not have yielded a better result. Inserting the actual value $q=1 / 2$ we obtain

$$
\frac{1}{2} \operatorname{tr}_{2}\left\{V \Gamma_{\rho}^{T}\right\} \geqq-\frac{3}{2 \pi}\left(\frac{4 \pi}{3}\right)^{4 / 3} \alpha^{2 / 3} \int M(z) M_{T}^{1 / 3}(z) d^{3} z
$$

We fix $0<\varepsilon \leqq 1 / 12$ and apply the Hölder and the maximal inequality (82) to the right hand of (87). This gives

$$
\begin{aligned}
\int M(z) M_{T}^{1 / 3}(z) d^{3} z \leqq & \left(\int[M(z)]^{\frac{3}{2+3 \varepsilon}} d^{3} z\right)^{2 / 3+\varepsilon}\left(\int\left[M_{T}(z)\right]^{\frac{1}{1-3 \varepsilon}} d^{3} z\right)^{1 / 3-\varepsilon} \\
\leqq & \left(\frac{96}{\pi}\right)\left(\frac{8}{3}\right)^{1 / 3} \varepsilon^{-1 / 3}\left(\int[\rho(x)]^{\frac{3}{2+3 \varepsilon}} d^{3} x\right)^{2 / 3+\varepsilon} \\
& \cdot\left(\int\left[\rho_{T}(x)\right]^{\frac{1}{1-3 \varepsilon}} d^{3} x\right)^{1 / 3-\varepsilon} .
\end{aligned}
$$


Applying the Hölder inequality again, we find

$$
\begin{gathered}
\int[\rho(x)]^{\frac{3}{2+3 \varepsilon}} d^{3} x \leqq\left(\int \rho^{5 / 3}(x) d^{3} x\right)^{\frac{3(1-3 \varepsilon)}{2(2+3 \varepsilon)}}\left(\int \rho(x) d^{3} x\right)^{\frac{1+15 \varepsilon}{2(2+3 \varepsilon)}} \\
\int\left[\rho_{T}(x)\right]^{\frac{1}{1-3 \varepsilon}} d^{3} x \leqq\left(\int \rho_{T}^{5 / 3}(x) d^{3} x\right)^{\frac{9 \varepsilon}{2(1-3 \varepsilon)}}\left(\int \rho_{T}(x) d^{3} x\right)^{\frac{2-15 \varepsilon}{2(1-3 \varepsilon)}} .
\end{gathered}
$$

We exploit $\rho_{T}^{5 / 3} \leqq \rho^{5 / 3}$ and get

$$
\begin{aligned}
& \left(\int[\rho(x)]^{\frac{3}{2+3 \varepsilon}} d^{3} x\right)^{2 / 3+\varepsilon}\left(\int\left[\rho_{T}(x)\right]^{\frac{1}{1-3 \varepsilon}} d^{3} x\right)^{1 / 3-\varepsilon} \\
& \leqq\left(\int \rho^{5 / 3}(x) d^{3} x\right)^{1 / 2}\left(\int \rho(x) d^{3} x\right)^{1 / 2}\left(\frac{\int \rho_{T}(x) d^{3} x}{\int \rho(x) d^{3} x}\right)^{1 / 3-5 \varepsilon / 2}
\end{aligned}
$$

Combining these estimates, we prove the assertion (78) above.

5.2. A Simple Bound on the Kinetic Energy. We need to bound the kinetic energy $\left\langle\Psi_{N}\left|\sum_{i=1}^{N}-\Delta_{i}\right| \Psi_{N}\right\rangle$ in order to prove Theorem 1. Our goal is to use as little information as possible about the system, i.e. about $\Psi_{N}, N, \underline{Z}$, and $\underline{R}$. We derive our bound essentially by reproducing a similar result of Lieb [10].

Lemma 6. Let $q \leqq N$ and $\Phi_{N} \in D_{N}$ such that $\left\langle\Phi_{N}\left|H_{N}(\underline{Z}, \underline{R})\right| \Phi_{N}\right\rangle \leqq 0$. Then

$$
\left\langle\Phi_{N}\left|\sum_{i=1}^{N}-\Delta_{i}\right| \Phi_{N}\right\rangle \leqq 2 q^{2 / 3} Z^{2} N^{1 / 3}
$$

Proof. Dropping the Coulomb interaction of the electrons, we have

$$
\begin{aligned}
0 \geqq & \left\langle\Phi_{N}\left|\sum_{i=1}^{N}\left(-\Delta_{i}-\sum_{j=1}^{K} \frac{Z_{j}}{\left|x_{i}-R_{j}\right|}\right)\right| \Phi_{N}\right\rangle \\
= & \frac{1}{2}\left\langle\Phi_{N}\left|\sum_{i=1}^{N}-\Delta_{i}\right| \Phi_{N}\right\rangle \\
& +\sum_{j=1}^{K} \frac{Z_{j}}{2 Z}\left\langle\Phi_{N}\left|\sum_{i=1}^{N}\left(-\Delta_{i}-\frac{2 Z}{\left|x_{i}-R_{j}\right|}\right)\right| \Phi_{N}\right\rangle \\
\geqq & \frac{1}{2}\left\langle\Phi_{N}\left|\sum_{i=1}^{N}-\Delta_{i}\right| \Phi_{N}\right\rangle-\sum_{j=1}^{K} \frac{Z_{j}}{2 Z} \frac{(2 Z)^{2}}{4} q\left(\frac{N}{q}\right)^{1 / 3} .
\end{aligned}
$$

In the last step we just added up the eigenvaues of the Bohr atom.

We are now in position to prove Theorem 1

Proof of Theorem 1. We recall the Lieb-Thirring inequality [15], which bounds $\int \rho^{5 / 3}$ by the kinetic energy. Namely, denoting $c_{\mathrm{LT}}:=2.7709 q^{-2 / 3}$ (see [9] for this value), it holds

$$
c_{\mathrm{LT}} \int \rho_{\Phi}^{5 / 3}(x) d^{3} x \leqq\left\langle\Phi_{N}\left|\sum_{i=1}^{N}-\Delta_{i}\right| \Phi_{N}\right\rangle,
$$


where $\rho_{\Phi}$ is the one-particle density of $\Phi_{N} \in D_{N}$. Inserting the Lieb-Thirring inequality into Lemmas 2 and 6 we thus complete the proof of Theorem 1.

\section{Asymptotics for Large $Z$}

Our goal in this section is to derive Theorem 2. The idea underlying our proof is that $\Psi_{N}$ is not only the ground state of $H_{N}(\underline{Z}, \underline{R})$, but it is an $\alpha$-approximate ground state of a suitable one-body Hamiltonian which approximates $H_{N}(\underline{Z}, \underline{R})$, too, where $\alpha=o\left(Z^{7 / 3}\right)$. However, the exact ground state $\Phi_{N}$, say, of such a one-body Hamiltonian is a Slater determinant and the corresponding 1-pdm is a projection. Therefore, we expect $\gamma$ to be close to a projection. It is here that we use semiclassical results, namely the considered one-body Hamiltonian is suggested by ThomasFermi theory.

We assume the existence of $\varepsilon, c_{1}, c_{2}, c_{3} \geqq 0$ such that

$$
\begin{array}{r}
\min \left\{\left|R_{i}-R_{j}\right| \mid 1 \leqq i<j \leqq K\right\} \geqq c_{1} Z^{-2 / 3+\varepsilon}, \\
Z-c_{2} Z^{1 / 3} \leqq N \leqq Z+c_{3} Z^{5 / 7},
\end{array}
$$

hold throughout this section without further notice.

6.1. The Semiclassical Results of Ivrii and Sigal. Let $\phi_{\mathrm{TF}}:=\phi_{\mathrm{TF}}(Z, \underline{Z}, \underline{R})$ be the Thomas-Fermi potential of a neutral molecule with nuclei $\underline{Z}$ at positions $\underline{R}$ (cf. [10]) and define

$$
H:=\left(-\Delta-\phi_{\mathrm{TF}}(x)\right) \otimes 1(\sigma)
$$

self-adjointly on $D \subseteq \mathscr{H}=L^{2}\left(\mathbb{R}^{3}\right) \otimes \mathbb{C}^{q}$. For all $\alpha>0$ we define the spectral projections $P_{\alpha}:=\chi_{(-\infty,-\alpha]}(H)$ and $P_{0}:=\chi_{(-\infty, 0)}(H)$. For $E \geqq 0$ we then denote

$$
H_{E}:=P_{E} H P_{E} \text {. }
$$

We define the energy $e \geqq 0$ as follows:

$$
-e:= \begin{cases}Z^{\text {th }} \text { eigenvalue of } H & \text { if } \operatorname{tr}\left\{P_{0}\right\}>Z \\ 0 & \text { if } \operatorname{tr}\left\{P_{0}\right\} \leqq Z\end{cases}
$$

In other words, if $H$ has at least $Z$ negative eigenvalues (counting multiplicities) then $-e$ is the $Z^{\text {th }}$ eigenvalue and otherwise $e=0$. Since $M:=\operatorname{tr}\left\{P_{e}\right\} \leqq Z, P_{e}$ certainly has an integral kernel and we define the corresponding one-particle density by $\rho_{M}(x):=\sum_{\sigma=1}^{q} P_{e}(x, \sigma \mid x, \sigma)$ on $\mathbb{R}^{3}$ a.e. Note that $\int \rho_{M}(x) d^{3} x=M$. Furthermore, we set for $f, g \in L^{1}\left(\mathbb{R}^{3}\right)$

$$
D(f, g):=\int \frac{d^{3} x d^{3} y}{|x-y|} f^{*}(x) g(y) .
$$

Let us quote the results of Ivrii and Sigal in form of a lemma (cf. [7], Thm. 2.4 and Lemma 2.8)

Lemma 7. (Ivrii and Sigal). Let $\min \left\{\left|R_{i}-R_{j}\right| \mid 1 \leqq i<j \leqq K\right\} \geqq \hat{c} Z^{-2 / 3+\varepsilon}$ for some $\hat{c}, \varepsilon>0$. Then, for some $c>0$ and for all $\alpha \geqq 0$,

(i) $D\left(\rho_{M}-\rho_{\mathrm{TF}}, \rho_{M}-\rho_{\mathrm{TF}}\right) \leqq c Z^{5 / 3}$,

(ii) $\operatorname{tr}\left\{P_{\alpha}\right\}=c_{s c} \int\left[\phi_{\mathrm{TF}}(x)-\alpha\right]_{+}^{3 / 2} d^{3} x+O\left(Z^{2 / 3}\right)$, where $c_{s c}:=6 \pi^{2} q^{-1}$. 
6.2. The Energy Truncation. Our first result in this section is an estimate on the energy expectation of $\gamma$ with respect to $H$. We claim

Lemma 8. Let $\gamma$ be the 1 -pdm of an E-approximate ground state of $H_{N}(\underline{Z}, \underline{R})$ with $\varepsilon \leqq \tilde{c} Z^{5 / 3}$. Then, for some $c \geqq 0$,

$$
\operatorname{tr}\{H \gamma\} \leqq \operatorname{tr}\left\{H_{e}\right\}+c Z^{5 / 3} .
$$

Proof. By the variational principle for $H_{M}(\underline{Z}, \underline{R})$ and the Lieb-Oxford inequality [13]

$$
\left\langle\Psi_{N}\left|\sum_{1 \leqq i<j \leqq N} \frac{1}{|x-y|}\right| \Psi_{N}\right\rangle \geqq \frac{1}{2} D(\rho, \rho)-(1.68) \int \rho^{4 / 3}(x) d^{3} x,
$$

we obtain

$$
\begin{aligned}
\operatorname{tr}\left\{H_{e}\right\}= & \operatorname{tr}\left\{h P_{e}\right\}+D\left(\rho_{\mathrm{TF}}, \rho_{M}\right) \\
= & \operatorname{tr}\left\{h P_{e}\right\}+\frac{1}{2} D\left(\rho_{M}, \rho_{M}\right)+\frac{1}{2} D\left(\rho_{\mathrm{TF}}, \rho_{\mathrm{TF}}\right) \\
& -\frac{1}{2} D\left(\rho_{M}-\rho_{\mathrm{TF}}, \rho_{M}-\rho_{\mathrm{TF}}\right) \\
\geqq & E_{Q}(M, \underline{Z}, \underline{R})+\frac{1}{2} D\left(\rho_{\mathrm{TF}}, \rho_{\mathrm{TF}}\right)-\frac{1}{2} D\left(\rho_{M}-\rho_{\mathrm{TF}}, \rho_{M}-\rho_{\mathrm{TF}}\right) \\
\geqq & \left.\operatorname{tr}\{h \gamma\}+\frac{1}{2} D(\rho, \rho)-\left[E_{Q}(N, \underline{Z}, \underline{R})\right]-E_{Q}(\underline{M}, \underline{Z}, \underline{R})\right]-\varepsilon \\
& -(1.68) \int \rho^{4 / 3}(x) d^{3} x+\frac{1}{2} D\left(\rho_{\mathrm{TF}}, \rho_{\mathrm{TF}}\right)-\frac{1}{2} D\left(\rho_{M}-\rho_{\mathrm{TF}}, \rho_{M}-\rho_{\mathrm{TF}}\right) \\
\geqq & \operatorname{tr}\{H \gamma\}-\left[E_{Q}(N, \underline{Z}, \underline{R})-E_{Q}(M, \underline{Z}, \underline{R})\right]-\varepsilon \\
& -(1.68) \int \rho^{4 / 3}(x) d^{3} x-\frac{1}{2} D\left(\rho_{M}-\rho_{\mathrm{TF}}, \rho_{M}-\rho_{\mathrm{TF}}\right) .
\end{aligned}
$$

Now, Lemma 7(i) states $D\left(\rho_{M}-\rho_{\mathrm{TF}}, \rho_{M}-\rho_{\mathrm{TF}}\right) \leqq c Z^{5 / 3}$ and using Lemma 6 yields

$$
\int \rho^{4 / 3}(x) d^{3} x \leqq\left(\int \rho^{5 / 3}(x) d^{3} x\right)^{1 / 2}\left(\int \rho(x) d^{3} x\right)^{1 / 2} \leqq c Z^{5 / 3} .
$$

Next, denoting $E_{N}:=E_{Q}(N, \underline{Z}, \underline{R})$, we show for all $N \geqq 3$

$$
\left|\frac{E_{N}}{N}\right| \leqq\left|\frac{E_{N-1}}{N-1}\right| \text {. }
$$

Namely, setting $h_{i}:=-\Delta_{i}+\sum_{j=1}^{K} Z_{j}\left|x_{i}-R_{j}\right|^{-1}$ and using an $\varepsilon^{\prime}$-approximate ground state $\Psi_{N} \in D_{N}$ of $H_{N}(\underline{Z}, \underline{R})$ for a suitably small $\varepsilon^{\prime}>0$, we infer

$$
\begin{aligned}
E_{N} \geqq & \left\langle\Psi_{N}\left|h_{1}+(N-1)\right| x_{1}-\left.x_{2}\right|^{-1} \mid \Psi_{N}\right\rangle-\varepsilon^{\prime} \\
& +\left\langle\Psi_{N}\left|\sum_{i=2}^{N} h_{i}+\sum_{2 \leqq i<j \leqq N} \frac{1}{\left|x_{i}-x_{j}\right|}\right| \Psi_{N}\right\rangle \\
\geqq & N^{-1} E_{N}+E_{N-1} .
\end{aligned}
$$


Now, if $N \geqq M$, we already arrive at the claim. Conversely assume $N<M$. Then, by (104),

$$
E_{N}-E_{M} \leqq(M-N)\left|\frac{E_{N}}{N}\right| \leqq c Z^{1 / 3} \cdot Z^{4 / 3} .
$$

We remark that $\operatorname{tr}\{H \gamma\}$ and $\operatorname{tr}\left\{H_{e}\right\}$ are both of order $Z^{7 / 3}$. Thus one may interpret Lemma 8 as stating that $\gamma$ cannot deviate much from $P_{e}=P_{e}^{2}$. It hence implicitly asserts the smallness of $\gamma-\gamma^{2}$. We introduce a cut-off in energy to make this explicit.

Lemma 9. Let $E>e$. Then, for some $c>0$,

$$
\operatorname{tr}\left\{P_{E}\left(\gamma-\gamma^{2}\right)\right\} \leqq c \frac{Z^{5 / 3}}{E}+\frac{e}{E} \operatorname{tr}\left\{P_{0}-P_{e}\right\}
$$

Proof. We observe that $0 \geqq \operatorname{tr}\left\{H_{0}\left(P_{e}-\gamma\right)^{2}\right\}$ implies $\operatorname{tr}\left\{\left(-H_{0}\right)\left(\gamma-\gamma^{2}\right)\right\}$ $\leqq \operatorname{tr}\left\{\left(-H_{e}\right)(1-\gamma)\right\}$. This inequality, $0 \leqq \gamma \leqq 1$, and $H_{0}-H \leqq 0 \leqq-H_{E}$ $\leqq-H_{e} \leqq-H_{0}$ yield

$$
\begin{aligned}
E \operatorname{tr}\left\{P_{E}\left(\gamma-\gamma^{2}\right)\right\} & \leqq \operatorname{tr}\left\{\left(-H_{E}\right)\left(\gamma-\gamma^{2}\right)\right\} \leqq \operatorname{tr}\left\{\left(-H_{e}\right)\left(\gamma-\gamma^{2}\right)\right\} \\
& \leqq \operatorname{tr}\left\{\left(-H_{e}\right)(1-\gamma)\right\} \\
& \leqq-\operatorname{tr}\left\{H_{e}\right\}+\operatorname{tr}\{H \gamma\}-\operatorname{tr}\left\{\left(H_{0}-H_{e}\right) \gamma\right\} \\
& \leqq c Z^{5 / 3}+e \operatorname{tr}\left\{P_{0}-P_{e}\right\},
\end{aligned}
$$

applying Lemma 8 in the last estimate.

As will be shown next, Lemma 8 does not only supply a bound on $\operatorname{tr}\left\{P_{E}\left(\gamma-\gamma^{2}\right)\right\}$, but also on $\operatorname{tr}\left\{\left(1-P_{E}\right)\left(\gamma-\gamma^{2}\right)\right\}$. Intuitively this is clear provided there are approximately $Z$ eigenvalues of $H$ below $-E$. More specifically, we will prove

Lemma 10. Let $E>e$. Then, for some $c>0$,

$$
\begin{aligned}
\operatorname{tr}\left\{\left(1-P_{E}\right)\left(\gamma-\gamma^{2}\right)\right\} \leqq & Z-\operatorname{tr}\left\{P_{E}\right\}+c Z^{5 / 7}+c \frac{Z^{5 / 3}}{E} \\
& +\frac{e}{E} \operatorname{tr}\left\{P_{0}-P_{e}\right\}
\end{aligned}
$$

Proof. As in the proof of Lemma 9 we observe

$$
E \operatorname{tr}\left\{P_{E}(1-\gamma)\right\} \leqq \operatorname{tr}\left\{\left(-H_{e}\right)(1-\gamma)\right\} \leqq c Z^{5 / 3}+e \operatorname{tr}\left\{P_{0}-P_{e}\right\}
$$

Also,

$$
\operatorname{tr}\left\{\left(1-P_{E}\right) \gamma\right\}=N-\operatorname{tr}\left\{P_{E}\right\}+\operatorname{tr}\left\{P_{E}(1-\gamma)\right\} .
$$

These two inequalities (110), (111) imply the assertion, since $\gamma \geqq \gamma-\gamma^{2}$ and $N \leqq Z+c Z^{5 / 7}$.

Now we will put these estimates together and insert the bound (ii) of Lemma 7 on $\operatorname{tr}\left\{P_{\alpha}\right\}, \alpha \geqq 0$. 
Lemma 11. Let $E>e$. Then, for some $c, c^{\prime}>0$,

$$
\operatorname{tr}\left\{\gamma-\gamma^{2}\right\} \leqq c Z^{5 / 7}+c^{\prime} \frac{Z^{5 / 3}}{E}+3 c_{s c} \int\left\{\phi_{\mathrm{TF}}^{3 / 2}(x)-\left[\phi_{\mathrm{TF}}(x)-E\right]_{+}^{3 / 2}\right\} d^{3} x .
$$

Proof. We add up the estimates from Lemma 9 and 10. This yields

$$
\operatorname{tr}\left\{\gamma-\gamma^{2}\right\} \leqq Z-\operatorname{tr}\left\{P_{E}\right\}+c \frac{Z^{5 / 3}}{E}+c_{1} Z^{5 / 7}+2 \frac{e}{E} \operatorname{tr}\left\{P_{0}-P_{e}\right\}
$$

By (ii) of Lemma 7 and Thomas-Fermi theory (cf. [11]) it holds

$$
Z=c_{s c} \int \phi_{\mathrm{TF}}^{3 / 2}(x) d^{3} x=\operatorname{tr}\left\{P_{0}\right\}+O\left(Z^{2 / 3}\right),
$$

which implies

$$
\frac{e}{E} \operatorname{tr}\left\{P_{0}-P_{e}\right\} \leqq Z-\operatorname{tr}\left\{P_{E}\right\}+c Z^{2 / 3}
$$

On the other hand, (ii) of Lemma 7 also asserts

$$
\operatorname{tr}\left\{P_{E}\right\} \geqq c_{s c} \int\left[\phi_{\mathrm{TF}}(x)-E\right]_{+}^{3 / 2} d^{3} x-c Z^{2 / 3},
$$

thus establishing Lemma 11.

6.3. The Semiclassical Number of Particles. As we have seen in the section before it remains to bound

$$
c_{s c} \int\left\{\phi_{\mathrm{TF}}^{3 / 2}(x)-\left[\phi_{\mathrm{TF}}(x)-E\right]_{+}^{3 / 2}\right\} d^{3} x
$$

and to assure $E>e$. We will do this by means of Lemma 12 below. Let us emphasize that the only parameters which enter in our estimate are $Z$ and $K$. Nothing needs to be assumed about $\underline{Z} / Z$ and $\underline{R}$.

Lemma 12. Let $\phi_{\mathrm{TF}}:=\phi_{\mathrm{TF}}(Z, \underline{Z}, \underline{R})$ be the Thomas-Fermi potential of a neutral molecule with nuclei $Z_{j}$ at positions $R_{j}$ for $1 \leqq j \leqq K$. Let furthermore $Z:=\sum_{j=1}^{K} Z_{j}$ and $0<E \leqq o\left(Z^{4 / 3}\right)$. Then, for sufficiently large $Z$,

$$
\begin{aligned}
\frac{2 \pi}{3}\left(\frac{3 c_{s c}}{\pi}\right)^{3 / 2} E^{3 / 4} & \leqq \int\left\{\phi_{\mathrm{TF}}^{3 / 2}(x)-\left[\phi_{\mathrm{TF}}(x)-E\right]_{+}^{3 / 2}\right\} d^{3} x \\
& \leqq \frac{16 \pi}{3} K^{7 / 4}\left(\frac{3 c_{s c}}{\pi}\right)^{3 / 2} E^{3 / 4}
\end{aligned}
$$

Proof. Let $\phi_{Z}$ be the Thomas-Fermi potential of a neutral atom at the origin. Using the maximum principle (cf. [11]) one sees that for all $1 \leqq j \leqq K$ and $x \in \mathbb{R}^{3}$ a.e.,

$$
\phi_{Z_{j}}\left(x-R_{j}\right) \leqq \phi_{\mathrm{TF}}(x) \leqq \sum_{j=1}^{K} \phi_{Z_{j}}\left(x-R_{j}\right) .
$$

We prove the inequality (118). Observe that $\phi^{3 / 2}-[\phi-E]_{+}^{3 / 2}$ is monotone in $\phi$. Since at least one of the nuclei, the $j^{\text {th }}$, say, has charge $Z_{j} \geqq Z / K$, we derive 


$$
\begin{gathered}
\phi_{\mathrm{TF}}(x) \geqq \phi_{Z_{j}}\left(x-R_{j}\right) \geqq \phi_{Z / K}\left(x-R_{j}\right), \text { and hence, using } \phi_{Z}(x)=Z^{4 / 3} \phi_{1}\left(Z^{1 / 3} x\right), \\
\int\left\{\phi_{\mathrm{TF}}^{3 / 2}(x)-\left[\phi_{\mathrm{TF}}(x)-E\right]_{+}^{3 / 2}\right\} d^{3} x \\
\geqq \int\left\{\phi_{Z / K}^{3 / 2}\left(x-R_{j}\right)-\left[\phi_{Z / K}\left(x-R_{j}\right)-E\right]_{+}^{3 / 2}\right\} d^{3} x \\
=\hat{Z} \int\left\{\phi_{1}^{3 / 2}(x)-\left[\phi_{1}(x)-\hat{E}\right]_{+}^{3 / 2}\right\} d^{3} x
\end{gathered}
$$

where $\hat{Z}:=Z / K$ and $\hat{E}:=E / \hat{Z}^{4 / 3}=o(1)$. As a result of Thomas-Fermi theory, $\phi_{1}(x)$ is a function of $|x|$ and decreases monotonically in $|x|$. Furthermore, $\phi_{1}(x) \leqq(3 / \pi)^{2} c_{s c}^{2}|x|^{-4}=: \tilde{c}^{2 / 3}|x|^{-4}$ and, indeed, for $|x|$ sufficiently large,

$$
2^{-2 / 3} \frac{\tilde{c}^{2 / 3}}{|x|^{4}} \leqq \phi_{1}(x) \leqq \frac{\tilde{c}^{2 / 3}}{|x|^{4}}
$$

(cf. [11]). Therefore, $\hat{E}=o(1)$ implies $\phi_{1}(x) \geqq \tilde{c} 2^{-2 / 3}|x|^{-4}$ on

$$
A:=\left\{x \in \mathbb{R}^{3} \mid \phi_{1}(x) \leqq E\right\} \supseteq\left\{\left.x \in \mathbb{R}^{3}\left|\tilde{c}^{2 / 3}\right| x\right|^{-4} \leqq E\right\}=: B
$$

This yields

$$
\begin{aligned}
\int\left\{\phi_{1}^{3 / 2}(x)-\left[\phi_{1}(x)-\hat{E}\right]_{+}^{3 / 2}\right\} d^{3} x & \geqq \int_{A} \phi_{1}^{3 / 2}(x) d^{3} x \geqq \int_{A} \frac{\tilde{c}}{2}|x|^{-6} d^{3} x \\
& \geqq \int_{B} \frac{\tilde{c}}{2}|x|^{-6} d^{3} x \\
& =4 \pi \frac{\tilde{c}}{2} \int_{\tilde{c}^{1 / 6} \hat{E}^{-1 / 4}}^{\infty} \frac{d r}{r^{4}}=\frac{4 \pi}{6} \tilde{c}^{1 / 2} \hat{E}^{3 / 4}
\end{aligned}
$$

Applying these estimates, the inequality (118) follows.

Now, we prove the inequality (119). We divide the space into the $K$ regions, $1 \leqq j \leqq K$,

$$
A_{j}:=\left\{x \in \mathbb{R}^{3}|\forall 1 \leqq i \leqq K:| x-R_{j}|\leqq| x-R_{i} \mid\right\} .
$$

On $A_{j}$, we estimate

$$
\varphi_{\mathrm{TF}}(x) \leqq \sum_{i=1}^{K} \phi_{Z_{i}}\left(x-R_{i}\right) \leqq \sum_{i=1}^{K} \phi_{Z_{\imath}}\left(x-R_{j}\right) \leqq K \phi_{Z}\left(x-R_{j}\right) .
$$

Hence it follows, with $\hat{E}:=E K^{-1} Z^{-4 / 3}$,

$$
\begin{aligned}
& \int\left\{\phi_{\mathrm{TF}}^{3 / 2}(x)-\left[\phi_{\mathrm{TF}}(x)-E\right]_{+}^{3 / 2}\right\} d^{3} x \\
& \quad \leqq \sum_{j=1}^{K} \int_{A_{j}}\left\{K^{3 / 2} \phi_{Z}^{3 / 2}\left(x-R_{j}\right)-\left[K \phi_{Z}\left(x-R_{j}\right)-E\right]_{+}^{3 / 2}\right\} d^{3} x \\
& \quad \leqq \sum_{j=1}^{K} \int\left\{K^{3 / 2} \phi_{Z}^{3 / 2}\left(x-R_{j}\right)-\left[K \phi_{Z}\left(x-R_{j}\right)-E\right]_{+}^{3 / 2}\right\} d^{3} x \\
& \quad=K^{5 / 2} Z \int\left\{\phi_{1}^{3 / 2}(x)-\left[\phi_{1}(x)-\hat{E}\right]_{+}^{3 / 2}\right\} d^{3} x
\end{aligned}
$$




$$
\begin{aligned}
& \leqq K^{5 / 2} Z \int\left\{\tilde{c}|x|^{-6}-\left[\tilde{c}^{2 / 3}|x|^{-4}-\hat{E}\right]_{+}^{3 / 2}\right\} d^{3} x \\
& \leqq 4 \pi K^{5 / 2} Z\left\{\hat{E} \int_{0}^{\tilde{c}^{1 / 6} \hat{E}^{-1 / 4}} \tilde{c}^{1 / 3} d r+\tilde{c} \int_{\tilde{c}^{1 / 6} \hat{E}^{-1 / 4}}^{\infty} \frac{d r}{r^{4}}\right\} \\
& =4 \pi K^{5 / 2} Z \frac{4 \tilde{c}^{1 / 2}}{3} \hat{E}^{3 / 4} .
\end{aligned}
$$

This establishes inequality (119) and hence Lemma 12.

We are now in position to prove Theorem 2

Proof of Theorem 2. In order to apply Lemma 11 we need to have an upper bound on $e$. First, assume that $e \geqq c Z$ for some $c>0$. Then $\operatorname{tr}\left\{P_{e}\right\}=Z$, by definition. Again applying (ii) of Lemma 7, this implies

$$
c_{s c} \int\left\{\phi_{\mathrm{TF}}^{3 / 2}(x)-\left[\phi_{\mathrm{TF}}(x)-e\right]_{+}^{3 / 2}\right\} d^{3} x \leqq Z-\operatorname{tr}\left\{P_{e}\right\}+c^{\prime} Z^{2 / 3} \leqq c^{\prime} Z^{2 / 3} .
$$

By the monotonicity in $e,(128)$ would still hold true if we replaced $e$ by $c Z$ on the left-hand side. Now, by Lemma 12 , we know that for $\bar{E}=o\left(Z^{4 / 3}\right)$,

$$
2\left(\frac{3}{\pi}\right)^{1 / 2} c_{s c}^{5 / 2} \bar{E}^{3 / 4} \leqq c_{s c} \int\left\{\phi_{\mathrm{TF}}^{3 / 2}(x)-\left[\phi_{\mathrm{TF}}(x)-\bar{E}\right]_{+}^{3 / 2}\right\} d^{3} x
$$

Hence, setting $\bar{E}:=c Z,(128)$ lead to the contradiction $Z^{3 / 4} \leqq$ const $Z^{2 / 3}$. Therefore, $e=o(Z) \leqq o\left(Z^{4 / 3}\right)$. This assures we may set $\bar{E}:=e$ in $(129)$ which, together with (128), implies

$$
e \leqq c Z^{8 / 9}
$$

Now, using Lemma 12, we derive from Lemma 11,

$$
\begin{aligned}
\operatorname{tr}\left\{\gamma-\gamma^{2}\right\} & \leqq c Z^{5 / 7}+c^{\prime} \frac{Z^{5 / 3}}{E}+3 c_{s c} \int\left\{\phi_{\mathrm{TF}}^{3 / 2}(x)-\left[\phi_{\mathrm{TF}}(x)-E\right]_{+}^{3 / 2}\right\} d^{3} x \\
& \leqq c Z^{5 / 7}+c^{\prime} \frac{Z^{5 / 3}}{E}+c^{\prime \prime} E^{3 / 4} \\
& \leqq c Z^{5 / 7}
\end{aligned}
$$

choosing $E:=Z^{20 / 21}=o\left(Z^{4 / 3}\right)$. This choice is justified for sufficiently large $Z$, since $E=Z^{20 / 21}>c Z^{8 / 9} \geqq e$.

We would like to emphasize that the method of proving Theorem 2 is not intrinsically semiclassical, despite the fact we use results from semiclassical analysis. Indeed, if we had substituted $H$ by some other one-body Hamiltonian $\tilde{H}$, say, we could have proved $\operatorname{tr}\left\{\gamma-\gamma^{2}\right\}=o(Z)$, provided its ground state energy agrees with $E_{Q}$ in leading order. Of course, we needed to have some explicit access to spectral functions of $\tilde{H}$ as well, which is why we chose $H=\left(-\Delta-\phi_{\mathrm{TF}}(x)\right) \otimes 1(\sigma)$. and comments. I also appreciate W. Hunziker's very constructive proofreading. Furthermore, I thank H. Siedentop, J.-P. Solovej, and M.B. Ruskai for various discussions and supplementary material. 


\section{References}

1. Coleman, A.J.: Structure of fermion density matrices. Rev. Mod. Phys. 35(3), 668-689 (1963)

2. Dirac, P.A.M.: Note on exchange phenomena in the Thomas-Fermi atom. Proc. Cambridge Philos. Soc. 26, 376-385 (1931)

3. Fefferman, C.L., de la Llave, R.: Relativistic stability of matter - I. Revista Matematica Iberoamericana, 2(1, 2), 119-161 (1986)

4. Fefferman, C.L., Seco, L.A.: The ground-state energy of a large atom. Bull. A.M.S. (1990)

5. Fefferman, C.L., Seco, L.A.: An upper bound for the number of electrons in a large ion. Proc. Nat. Acad. Sci. USA, 86, 3464-3465 (1989)

6. Hughes, W.: An Atomic Energy Lower Bound that Gives Scott's Correction. PhD thesis, Princeton, Department of Mathematics, 1986

7. Ivrii, V.Ja., Sigal, I.M.: Asymptotics of the ground state energies of large Coulomb systems. Annals Math. (to appear)

8. Lieb, E.H.: A lower bound for Coulomb energies. Phys. Lett. 70A, 444-446 (1979)

9. Lieb, E.H.: On characteristic exponents in turbulence. Commun. Math. Phys. 92, 473-480 (1984)

10. Lieb, E.H.: The stability of matter. Rev. Mod. Phys. 48, 653-669 (1976)

11. Lieb, E.H.: Thomas-Fermi and related theories of atoms and molecules. Rev. Mod. Phys. 53, 603-604 (1981)

12. Lieb, E.H.: Variational principle for many-fermion systems. Phys. Rev. Lett. 46(7), 457-459 (1981)

13. Lieb, E.H., Oxford, S.: An improved lower bound on the indirect Coulomb energy. Int. J. Quantum Chem. 19, 427-439 (1981)

14. Lieb, E.H., Simon, B.: The Thomas-Fermi theory of atoms, molecules and solids. Adv. Math. 23, 22-116 (1977)

15. Lieb, E.H., Thirring, W.E.: Inequalities for the moments of the eigenvalues of the Schrödinger Hamiltonian and their relation to Sobolev inequalities. In Lieb, E.H., Simon, B., Wightman, A.S. (eds.), Studies in Mathematical Physics: Essays in Honor of Valentine Bargmann. Princeton, NJ: Princeton University Press 1976

16. Schwinger, J.: Thomas-Fermi model: The second correction. Phys. Rev. A 24(5), 2353-2361 (1981)

17. Siedentop, H.K.H., Weikard, R.: A new phase space localization technique with application to the sum of negative eigenvalues of Schrödinger operators. Ann. Scient. Ec. Norm. Sup. 24, 215-225 (1991)

18. Siedentop, H.K.H., Weikard, R.: On the leading energy correction for the statistical model of the atom: Interacting case. Commun. Math. Phys. 112, 471-490 (1987)

19. Stein, E.M., Weiss, G.: Introduction to Fourier Analysis on Euclidean Spaces. Princeton, New Jersey: Princeton University Press, 2 edition, 1971

20. Thirring, W.: Lehrbuch der Mathematischen Physik 4: Quantenmechanik großer Systeme. Wien, New York: Springer, 1 edition, 1980

Communicated by B. Simon 